\title{
Development of a First-in-Class Small Molecule Inhibitor of the C-terminal Hsp90 Dimerization
}

Sanil Bhatia ${ }^{{ }^{*}}$, Lukas Spanier ${ }^{2 *}$, David Bickel ${ }^{2}$, Niklas Dienstbier $^{1}$, Vitalij Woloschin ${ }^{2}$, Melina Vogt ${ }^{1}$, Henrik Pols, ${ }^{2}$ Beate Lungerich, ${ }^{2}$ Jens Reiners ${ }^{3}$, Narges Aghaallaei ${ }^{4}$, Daniela Diedrich ${ }^{2}$, Benedikt Frieg ${ }^{2,5}$, Julian Schliehe-Diecks ${ }^{1}$, Bertan Bopp ${ }^{6}$, Franziska Lang ${ }^{1}$, Mohanraj Gopalswamy ${ }^{2}$, Jennifer Loschwitz ${ }^{2}$, Baubak Bajohgli ${ }^{4}$, Julia Skokowa ${ }^{4}$, Arndt Borkhardt ${ }^{1}$, Julia Hauer ${ }^{7,8}$, Finn K. Hansen ${ }^{9}$, Sander H.J. Smits $^{3,10}$, Joachim Jose ${ }^{6}$, Holger Gohlke ${ }^{2,5 \#}$ and Thomas Kurz ${ }^{2 \#}$

\section{Affiliations:}

1. Department of Pediatric Oncology, Hematology and Clinical Immunology, Medical Faculty, Heinrich Heine University Düsseldorf, Düsseldorf, Germany

2. Institute for Pharmaceutical and Medicinal Chemistry, Heinrich Heine University Düsseldorf, Düsseldorf, Germany

3. Center for Structural Studies, Heinrich Heine University Düsseldorf, Düsseldorf, Germany

4. Department of Hematology, Oncology, Clinical Immunology and Rheumatology, University Hospital Tübingen, Germany

5. John von Neumann Institute for Computing (NIC), Jülich Supercomputing Centre (JSC), Institute of Biological Information Processing (IBI-7: Structural Biochemistry) \& Institute of Bio- and Geosciences (IBG-4: Bioinformatics), Forschungszentrum Jülich GmbH, Jülich, Germany

6. Institute for Pharmaceutical and Medicinal Chemistry, PharmaCampus, Westphalian Wilhelms University, Münster, Germany

7. Department of Pediatrics, Pediatric Hematology and Oncology, University Hospital Carl Gustav Carus, Dresden, Germany

8. National Center for Tumor Diseases (NCT), Partner Site Dresden, Dresden, Germany

9. Pharmaceutical and Cell Biological Chemistry, Pharmaceutical Institute University of Bonn, Bonn, Germany

10. Institute of Biochemistry, Heinrich Heine University Düsseldorf, Düsseldorf, Germany

* contributed equally to this work

\# shared senior authorship

\# Corresponding authors: (T.K.) Universitätsstr. 1, 40225 Düsseldorf, Germany, Phone: (+49) 21181 14984, E-mail: thomas.kurz@uni-duesseldorf.de; (H.G.) Universitätsstr. 1, 40225 Düsseldorf, Germany, Phone: (+49) 21181 13662, E-mail: gohlke@uni-dueseldorf.de; (S.B.) Moorenstraße 5, 40225 Düsseldorf, Germany, Phone (+49) 2118104896 Email, sanil.bhatia@med.uni-duesseldorf.de 
Author ORCID: Benedikt Frieg: 0000-0002-7877-0262; Holger Gohlke: 0000-0001-8613-1447; David Bickel: 0000-0003-0332-8338; Sanil Bhatia: 0000-0001-6494-7744; Finn K. Hansen: 00000001-9765-5975 Thomas Kurz: 0000-0002-9474-4224; Sander Smits 0000-0003-0780-9251; Baubak Bajoghli: 0000-0002-7368-7523; Jennifer Loschwitz: 0000-0002-9066-1923

Keywords: protein-protein interaction, heat-shock protein, CML, BCR-ABL1, imatinib, Hsp90 inhibitor. 


\section{Abstract}

Heat shock protein 90 (Hsp90) is a promising therapeutic target due to its involvement in stabilizing several aberrantly expressed oncoproteins. In cancerous cells, Hsp90 expression is elevated, thereby contributing in exerting anti-apoptotic effects, which is essential for the malignant transformation and progression of several tumor types. Most of the Hsp90 inhibitors (Hsp90i) under investigation target the ATP binding site in the N-terminal domain (NTD) of Hsp90. However, adverse effects, including induction of the pro-survival resistance mechanism (heat shock response or HSR) and associated doselimiting toxicity, have so far precluded clinical approval of these Hsp90i. In contrast, modulators that interfere with the C-terminal domain (CTD) of Hsp90 do not inflict HSR and, thus, emerge as a promising alternative approach to target Hsp90. Since the CTD dimerization of Hsp90 is essential for its chaperone activity, interfering with this essential dimerization process by small-molecule proteinprotein interaction (PPI) inhibitors is a promising strategy for anticancer drug research. We have developed the first-in-class small molecule inhibitor (5b) targeting the Hsp90 CTD dimerization interface, based on a tripyrimidonamide scaffold through structure-based molecular design, chemical synthesis, binding mode model prediction, assessment of the biochemical affinity and efficacy against therapy-resistant leukemia cells. $\mathbf{5 b}$ reduces xenotransplantation of leukemia cells in zebrafish models and induces apoptosis in BCR-ABL1+(T315I) tyrosine kinase inhibitors (TKIs) resistant leukemia cells, without inducing HSR. 


\section{Introduction}

The heat shock protein (Hsp90) is an abundant, cytosolic molecular chaperone that modulates the folding, stabilization, and maturation of over 400 client proteins in eukaryotes. ${ }^{1}$ It is involved in essential processes such as signal transduction, cell cycle progression, and transcription regulation. ${ }^{1}$ In cancer cells, Hsp90 is overexpressed, involved in uncontrolled proliferation and anti-apoptotic effects, and, that way, essential for the malignant transformation and progression of several cancer types. ${ }^{2}$ Thus, cancer cells are more dependent on Hsp90 activity than normal cells. ${ }^{3,4}$ In various cancers, multiple signal transduction-promoting oncoproteins are clients of Hsp90. ${ }^{5}$ Hence, inhibiting the activity of Hsp90 is a promising strategy for the development of anticancer therapy. Several Hsp90 inhibitors (Hsp90i) have been developed recently, however adverse effects including dose-limiting ocular and cardiac toxicity and poor patient stratification have precluded their clinical approval. ${ }^{3}$ Most of the 19 Hsp90 inhibitors (Hsp90i) studied in clinical trials so far (except RTA 901) target the N-terminal ATP binding site (NTD) and are termed N-terminal inhibitors. ${ }^{3,6-15}$ A common pitfall of Hsp90 NTD-targeting inhibitors is the induction of a pro-survival heat shock response (HSR). ${ }^{3,6}$ The HSR is a stress response mechanism mediated by heat-shock factor 1 (HSF-1), which leads to the expression of other heat shock proteins (HSPs) including Hsp27, Hsp40 and Hsp70 as a rescue mechanism upon Hsp90 inhibition and eventually weakens cytotoxic effects of Hsp90i. ${ }^{3,6,11-14}$ In addition, Hsp90 NTD-targeting inhibitors potentially inflict cytotoxicity through mechanisms that involve targets other than Hsp90, (off-target effects). ${ }^{4,16}$ The off-target effect hypothesis is also supported by the drastic differences between cytotoxicity concentrations of Hsp90 NTD-targeting inhibitors vs. their binding affinity to Hsp90. ${ }^{16}$ Furthermore, there are two major cytosolic isoforms of Hsp90 (Hsp90 $\alpha$ and Hsp90ß) expressed in humans. Hsp90 $\alpha$ is an inducible isoform, overexpressed in several cancer types, whereas the Hsp90 $\beta$ isoform is expressed constitutively. Thus, targeting Hsp90 with isoform-specific inhibitors can afford a therapeutic window. ${ }^{17,18}$ Most of the previously studied Hsp90i exhibit pan-inhibitory activity (i.e., targeting all isoforms). However, the Hsp90 $\alpha$ and Hsp90 $\beta$ isoforms share a high degree of similarity (SI Figure S21), making it challenging to develop isoform-selective inhibitors. ${ }^{18}$ 
Hsp90 is a flexible homodimer, and each monomer consists of three major functional domains: Nterminal domain (NTD), middle domain, and C-terminal domain (CTD). Hsp90's activity depends on the binding and hydrolysis of ATP at the NTD and on its dimerization via the CTD. ${ }^{2}$ The middle domain (MD) that connects the NTD and the CTD mediates the binding of clients and cochaperons. The CTD is connected to the MEEVD motif, which interacts with the subset of tetratricopeptide repeat (TPR) domain-containing cochaperones. ${ }^{2}$ To our knowledge, inhibiting Hsp90 dimer formation by targeting the CTD dimerization interface constitutes a so far unexplored mode of action (MOA) of small-molecule Hsp90i. In contrast to Hsp90i targeting the N-terminal ATP binding site, C-terminal inhibitors do not generally induce HSR. ${ }^{6,16,19-21}$ The most important classes of C-terminal inhibitors are: (1) inhibitors binding to the C-terminal ATP-binding site (e.g., novobiocin and analogs), (2) modulators of the Hsp90CDC37 interaction (e.g., celastrol, induces HSR ${ }^{22}$ ), (3) modulators of the Hsp90-p23 interaction (e.g., gedunin), (4) modulators of the Hsp90-HOP interaction (e.g., LB76), and (5) aminoxyrone (AX), the first non-peptidic inhibitor of the C-terminal dimerization of $\mathrm{Hsp} 90 .^{23}$

Following a strategy recently introduced by us to identify PPI inhibitors, ${ }^{24,25}$ we initially identified hot spot residues in the CTD dimerization interface that accounted for most of the binding affinity ${ }^{26}$ and identified the first peptidic inhibitors shown to bind to the CTD of Hsp90. ${ }^{27}$ Furthermore, we developed AX, the first peptidomimetic Hsp90 CTD dimerization inhibitor, ${ }^{28}$ which is a promising lead candidate effective against BCR-ABL1+ TKI-resistant leukemic cells. ${ }^{28}$ Based on these experiences, here, we report the rational design, chemical synthesis, binding mode model, biochemical affinity, and biological in vitro evaluation of the first-in-class small molecule inhibitor (5b) of Hsp90 CTD dimerization based on a tripyrimidonamide scaffold.

\section{Results}

\section{Design of tripyrimidonamides as CTD Hsp 90 inhibitors}

Based on computational predictions and subsequent experimental validation, we identified the spatially clustered hot spot residues I688, Y689, I692, and L696 in the Hsp90 CTD interface, which are located on $\alpha$-helix H5, form a functional epitope, and account for most of the protein dimerization energy. ${ }^{26}$ 
Furthermore, conformational analysis by $2 \mathrm{D}$ NMR and MD simulations revealed for the recently introduced tripyrimidonamide scaffold that it can act as a potential $\alpha$-helix mimetic, mimicking side chains at positions $i, i+4$ (dimeric compound) or $i, i+4, i+8$ (trimeric compound). ${ }^{29,30}$ This side chain pattern is concordant with the succession of the hot spot residues in the Hsp90 CTD interface.

Together, this provided the incentive for us to design and synthesize the tripyrimidonamide 5a, which mimics the hot spots I688, I692, and L696. In compound 5a, the side chain of V was used instead of I to avoid diasteromeres. 6, which lacks the isopropyl side chain, was also designed to probe the influence of the absence of the third side chain in a tripyrimidonamide. Next, we aimed to design compounds that can also form polar interactions, as these should confer specificity of binding. ${ }^{26}$ In addition, the binding to a well-defined cleft or groove in a PPI region has been described to yield a particularly effective PPI inhibitor. ${ }^{26}$ The Y689 side chain of 7a should be accommodated in an indentation in the binding epitope of helix H4' (Figure 1); ${ }^{26}$ we also designed the homolog $\mathbf{7 b}$ with a prolonged (4-hydroxy-phenyl)-ethyl side chain. Although both compounds mimic the three hot spots Y689, I692, L696, with the longer side chain in $\mathbf{7 b}$, we intended to accommodate for the apparent mismatch between the preferred side chain orientations in tripyrimidonamides and the side chain pattern of the hot spots $(i, i+3, i+4)$. The side chain patterns of $\mathbf{5 b}$ and $7 \mathbf{a}$ are almost identical to that of the $\alpha$-aminoxy-peptide AX, which was shown to bind to the CTD. ${ }^{28}$

Further analysis of the physicochemical properties of the CTD dimerization interface revealed a particular hydrophobic patch there (Figure 1). Interestingly, the 4-methoxy-benzyl side chain of $\mathbf{5 b}$ should act as a (weak) hydrogen bond acceptor for S673' and T669' on helix H4', but at the same time decrease the side chain's hydrophilicity for a more favorable burial in the overall hydrophobic interface. To probe this with a larger substituent, we also designed the benzyloxy derivatives $\mathbf{5 c}$ and $\mathbf{5 d}$, respectively, which are also precursors of $\mathbf{7 a}$ and $\mathbf{7 b}$.

\section{Synthesis of tripyrimidonamides}

The monomeric building blocks $\mathbf{1}$ and 2a-e were prepared according to our previously published protocol. ${ }^{30}$ Subsequently, the designed tripyrimidonamides 5a-d were synthesized using a modular approach. Briefly, a COMU-mediated amide coupling of the lithium carboxylate 2e with 
5-aminopyrimidone 1 afforded the benzoyl-protected dimer 3 in $75 \%$ yield. Deprotection of the benzoylgroup by treatment of $\mathbf{3}$ with sodium hydroxide in methanol at $80^{\circ} \mathrm{C}$ afforded the unprotected dimer 4 (77\% yield). Additional coupling reactions of $\mathbf{4}$ with the respective lithium salts $\mathbf{2 a - d}$ in the presence of COMU furnished the tripyrimidonamides 5a-d in 39-76\% yield. Compound $\mathbf{6}$ with an $N$-unsubstituted N-terminal pyrimidone ring was synthesized by treating the corresponding 4-methoxybenzyl-substituted derivative $\mathbf{5 b}$ with $\mathrm{BBr}_{3}$ in $\mathrm{DCM}$ (Scheme 1). Finally, the trispyrimidonamides $\mathbf{7 a}$ and $\mathbf{7 b}$ with free phenolic groups were prepared by catalytic hydrogenation of their respective $O$-benzyl-protected precursors $\mathbf{5 c}$ and $\mathbf{5 d}$ (40 and $87 \%$ yield). 


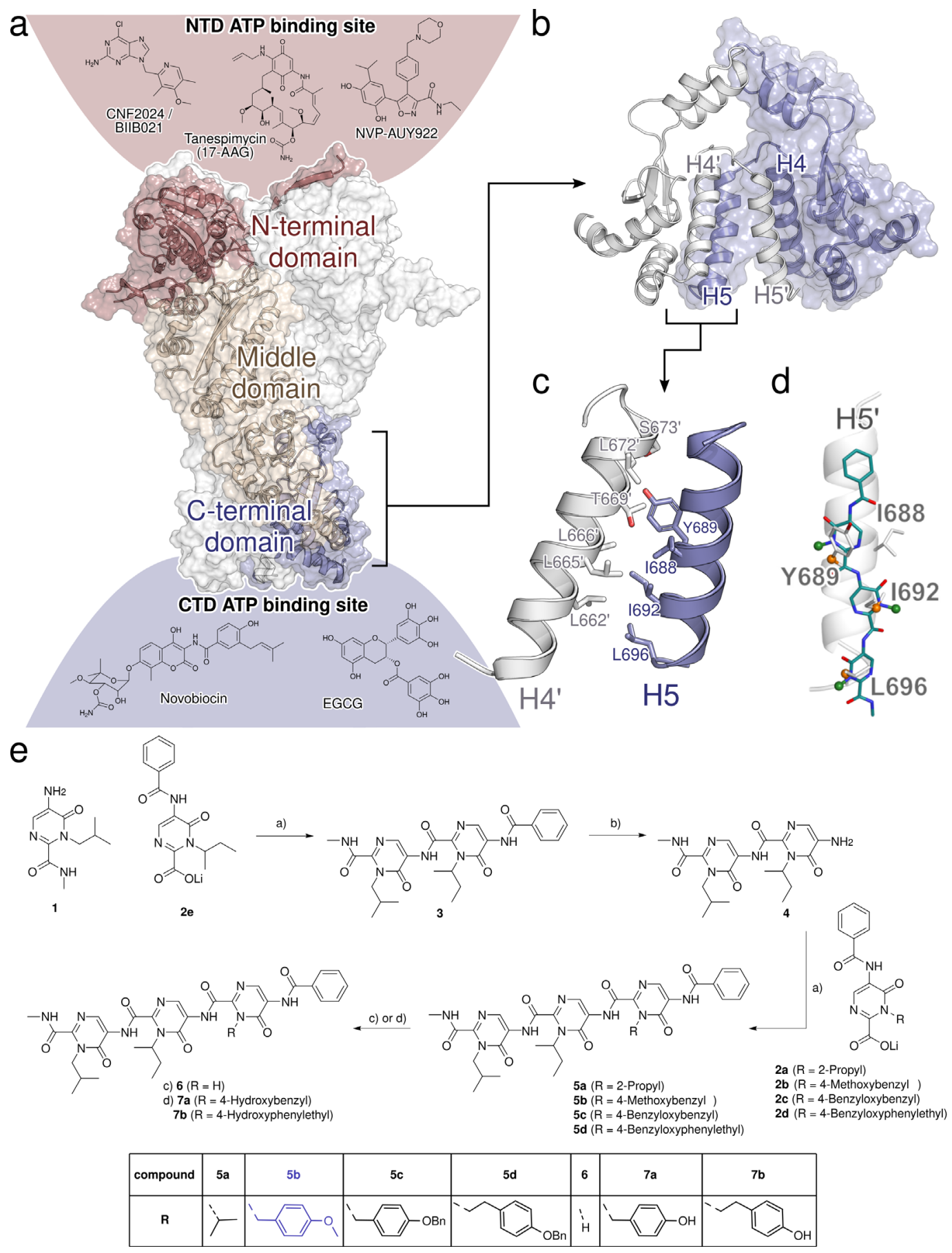

Figure 1: Rational design and synthesis of tripyrimidoneamides. a) Cryo-EM structure of the dimer of human Hsp90 3 (PDB ID 5FWK), ${ }^{31}$ shown in surface and cartoon representation. For one of the Hsp90 monomers, the Nterminal domain (NTD) is colored in red, the middle domain in beige, and the C-terminal domain (CTD) in blue. Above and below the protein structure, the structures of Hsp90i and their potential binding sites (see ref. ${ }^{32-36}$, color-coded according to the domains) are shown. b) Dimeric CTD of human Hsp90 $\beta$ with the two monomers in blue and white. Helices H4, H4', H5, and H5' of the CTDs form the dimerization interface. c) Residues forming the CTDs dimerization interface in human Hsp90 $\alpha$ are primarily located on helices H4, H4', H5, and H5 '. ${ }^{26}$ d) Trispyrimidones can adopt conformations resembling the side chain orientation of an $\alpha$-helix in $i, i+4$ and $i+7$ position. ${ }^{30}$ e) Synthesis of tripyrimidoneamides: a) COMU, DMF, r. t., 18 h; b) $\mathrm{NaOH}, \mathrm{MeOH}, 80{ }^{\circ} \mathrm{C}, 6 \mathrm{~h}$; a) 2 ad, COMU, DMF, r. t., 18 h; c) 6 via 5b, $\mathrm{BBr}_{3}, \mathrm{DCM},-78{ }^{\circ} \mathrm{C}, 1 \mathrm{~h}, \mathrm{r} . \mathrm{t}$., $1 \mathrm{~h}$; d) via $7 \mathbf{a}$ via $\mathbf{5 c}$ and $7 \mathbf{b}$ via $\mathbf{5 d}, \mathrm{H}_{2}$, $\mathrm{Pd}(\mathrm{C}), \mathrm{MeOH}, \mathrm{DCM}$, r. t., $1 \mathrm{~h}$. 


\section{Selection of $\mathbf{5 b}$ as a lead candidate}

To evaluate the inhibition of Hsp90 dimerization, E. coli BL21 (DE3) pETSH-3 cells were used to display Hsp90 $\alpha$ on their surface (Figure 2a). ${ }^{27}$ Passenger-driven dimer formation of Hsp90 $\alpha$ is facilitated through the motility of the $\beta$-barrel domain within the outer membrane of $E$. coli, as reported for other proteins. ${ }^{37}$ To demonstrate the functionality of dimerized Hsp90 on the surface of E. coli, the transcription factor $\mathrm{p} 53$, a natural client protein of Hsp90, was labeled with fluorescein isothiocyanate (FITC) and added to cells displaying Hsp90 on their surface. Subsequent flow cytometer analysis revealed a high green fluorescence for cells displaying Hsp90, indicating dimerized and functional Hsp90 (Figure 2b). Compounds 5a, 6, and 7a showed only weak inhibition of $3.27 \%, 14.65 \%$, and $24.35 \%$, respectively. In contrast, $\mathbf{5 b}, \mathbf{5 c}, \mathbf{5 d}$, and $\mathbf{7 b}$ showed moderate inhibition of $39.92 \%, 41.83 \%$, $55.23 \%$, and $31.33 \%$, respectively (Figure $2 \mathbf{b}$ ).

Later, the binding affinity of the compounds was determined with microscale thermophoresis (MST) measurements, using NT-647-labeled recombinant CTD of Hsp90 $\alpha$ protein. ${ }^{28}$ A nonlinear regression curve was fitted with the $K_{\mathrm{D}}$ formula, and, as expected, substances showing weak inhibition have high dissociation constants (6: $249 \mu \mathrm{M}, 7 \mathbf{a}: 286 \mu \mathrm{M}$; Figure 2c, e). The lowest $K_{\mathrm{D}}$ value was observed for 5b with $3.42 \mu \mathrm{M}$ (Figure 2c, e). Next, the in vitro cytotoxicity assessment of compounds 5a-d, 6, 7a, and $\mathbf{7 b}$ revealed $\mathbf{5 b}$ as a promising candidate (with low $\mathrm{IC}_{50}: 1.8 \pm 0.3 \mu \mathrm{M}$ ) in a BCR-ABL1+ tested leukemia cell line K562 (Figure 2d, 2e).

Based on the inhibition of Hsp90 $\alpha$ CTD dimerization, low apparent $K_{\mathrm{D}}$ value for the Hsp90 $\alpha$ CTD, and potent anti-leukemic activity, $\mathbf{5 b}$ was selected for further detailed affinity and efficacy assessments. 
a

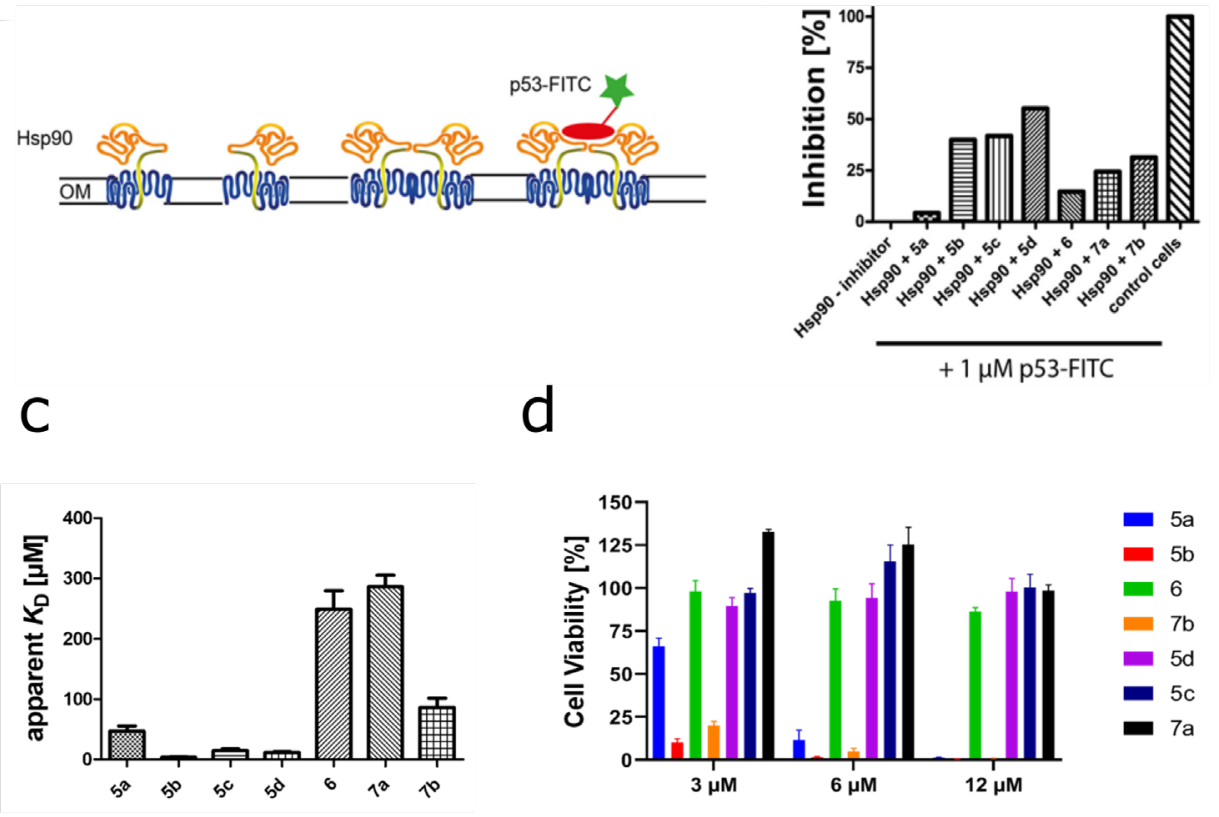

e

\begin{tabular}{|c|c|c|c|}
\hline Inhibitor & $\begin{array}{c}\text { Inhibition (\%) of } \\
\text { Hsp90 dimerization } \\
\text { at } 50 \mu \mathrm{M}\end{array}$ & $\begin{array}{l}\text { Apparent } K_{D} \\
\text { versus } \\
\text { Hsp90a CTD }\end{array}$ & $\begin{array}{c}\mathrm{K} 562 \\
\left(\mathrm{IC}_{50} \mu \mathrm{M}\right)\end{array}$ \\
\hline $5 a$ & 3.27 & $47.2 \pm 8.6$ & $3.5 \pm 0.1$ \\
\hline $5 b$ & 39.9 & $3.4 \pm 1.0$ & $1.3 \pm 0.3$ \\
\hline $5 c$ & 41.8 & $14.9 \pm 2.4$ & $66.8 \pm 13.0$ \\
\hline $5 d$ & 55.2 & $11.7 \pm 1.0$ & $97.9 \pm 17.2$ \\
\hline 6 & 14.6 & $249 \pm 30.7$ & $100.3 \pm 25$ \\
\hline $7 a$ & 24.3 & $286 \pm 19.5$ & $60.8 \pm 23.1$ \\
\hline $7 \mathrm{~b}$ & 31.3 & $86.4 \pm 15$ & $1.6 \pm 0.1$ \\
\hline
\end{tabular}

Figure 2: Selection of $\mathbf{5 b}$ as a lead candidate. a) Schematic view of the Hsp90 dimerization assay using Autodisplay. b) Flow cytometry measurements of the inhibition of dimerized Hsp90 $\alpha$ displayed on E. coli cells. ${ }^{28}$ E. coli BL21 (DE3) cells displaying Hsp90 $\alpha$ incubated with $1 \mu \mathrm{M}$ FITC-labeled p53 lead to a high cellular fluorescence indicating dimerization of $\mathrm{Hsp} 90 \alpha$. The value obtained was set as $0 \%$ inhibition. In contrast, E. coli cells without displaying Hsp90 $\alpha$ (control cells) show no cellular fluorescence. The value obtained here was set as $100 \%$ inhibition. Preincubation of E. coli cells with surface-displayed Hsp90 $\alpha$ with $50 \mu \mathrm{M}$ of the respective substance leads to a lowered cellular fluorescence intensity indicating a lowered binding affinity of FITC-labeled p53 to surface-displayed Hsp90 $\alpha$. These values were set in relation to obtain the relative inhibition of dimerization. c) Apparent $K_{\mathrm{D}}$ values of purified CTD of Hsp90 $\alpha$ and the respective substance measured via the MST method. A constant amount of $50 \mathrm{nM}$ labeled CTD of Hsp90 was used, and three independent measurements were performed. The resulting mean values were determined and used in the $K_{\mathrm{D}}$ Fit formula. d) Cellular viability assessment of a leukemic cell line (K562) measured by incubating with the indicated inhibitors for $72 \mathrm{~h}$, followed by viability measurement using ATP-based Celltitre Glo assay. e) Selection of $\mathbf{5 b}$ as a lead candidate on the basis high inhibition of Hsp90 $\alpha$ dimerization, low apparent $K_{\mathrm{D}}$, and low $\mathrm{IC}_{50}(\mu \mathrm{M})$ in a tested leukemic cell line. 


\section{$5 b$ binds specifically to CTD of Hsp90 $\alpha$ and blocks it cochaperone function}

One of the major limitations of NTD-targeting inhibitors is their off-target activity. ${ }^{4,16}$ Hence, it is important that the selected hit $\mathbf{5 b}$ has a high degree of selectivity against its target, the CTD of Hsp90. To assess the selectivity of $\mathbf{5 b}$, biochemical cell-free and cellular assays were performed. First, we evaluated the affinity of $\mathbf{5 b}$ against Hsp90 in a cell-free assay, where $\mathbf{5 b}$ protected recombinant (fulllength) Hsp90a protein in a dose-dependent fashion from degradation against thermolysin enzyme digestion, an assay commonly used to quantify drug affinity-responsive target stability (DARTS) $)^{19,38}$ (Figure 3a). Next, we performed the cell-free thermal shift assay ${ }^{39}$ to determine the potential binding affinity of $\mathbf{5 b}$ to recombinant Hsp90 $\alpha$ CTD and NTD protein. $\mathbf{5 b}$ specifically destabilized the CTD of Hsp90 $\alpha$ protein $\mathrm{Hsp} 90 \mathrm{CTD}\left(\Delta \mathrm{Tm}:-8.45 \pm 0.55{ }^{\circ} \mathrm{C}\right)$, whereas reference CTD targeting Hsp90i coumermycin A1 (CA1) stabilized Hsp90 $\alpha$ CTD protein (Figure 3b, SI Figure 22 and SI Table 1). NTD targeting Hsp90i Tanespimycin (TM) and PUH-71 served as positive (Hsp90 $\alpha$ NTD) or negative control (Hsp90 $\alpha$ CTD) in this assay. The thermostabilizing effect of $\mathbf{5 b}$ to its target (total Hsp90) was also assessed in a cellular setup, termed cellular thermal shift assay (CETSA) $)^{39-41}$, a biophysical method based on the ligand-induced thermal stabilization of the protein to directly probe the target engagement in the living cells (SI Table 1, SI Figure 23). The protein quantification for CETSA was performed using a digital western blotter for sensitive and quantitative evaluation of the ligand-protected intracellular Hsp90, whereas TM and PU-H71 served as controls. Next, the thermal stability of intracellular Hsp90 in an increasing concentration of $\mathbf{5 b}$ (at a fixed temperature) was determined, a method termed isothermal dose-response fingerprint $\operatorname{ITDRF}_{\mathrm{CETSA}} \cdot{ }^{40} \mathbf{5 b}$ induced thermal stability of Hsp90 in a dose-dependent fashion, confirming its intracellular and specific target engagement (Figure

\section{3c, SI Table 1).}

Next, to assess the ability of $\mathbf{5 b}$ to inhibit Hsp90 chaperone function, a cell-free luciferase-refolding assay $^{42-44}$ was performed using rabbit reticulocyte lysates as a source of Hsp90. Exposure of $\mathbf{5 b}$ decreased the luciferase refolding capacity in a dose-dependent manner by blocking the chaperone function of Hsp90 (Figure 3d). The known Hsp90 NTD inhibitors geldanamycin (GM) and TM served as positive controls. Besides, to assess the specific effect of $\mathbf{5 b}$ in obstructing Hsp90 CTD-interacting cochaperones, a time-resolved fluorescence resonance energy transfer (TR-FRET) assay was 
conducted. ${ }^{45} \mathbf{5 b}$ blocked the binding of PPID (or cyclophillin D, an Hsp90 CTD-interacting chaperone) to recombinant Hsp90 $\alpha$ or Hsp90 $\beta$ CTD protein comparable to CA1 treatment, whereas PU-H71, TM, and GM served as negative controls (Figure 3e, Table 1). To rule out the possible interaction of $\mathbf{5 b}$ with the NTD of Hsp90 $\alpha$, a fluorescence polarization (FP) competitive assay was carried out using FITClabelled $\mathrm{GM}^{42}$ (Figure 3f, SI Table 1). As expected, 5b did not show any interaction with the NTD of Hsp90, whereas unlabeled Hsp90 NTD targeting inhibitors GM, Ganetespib (GP), TM, and PU-H71 served as positive controls. 

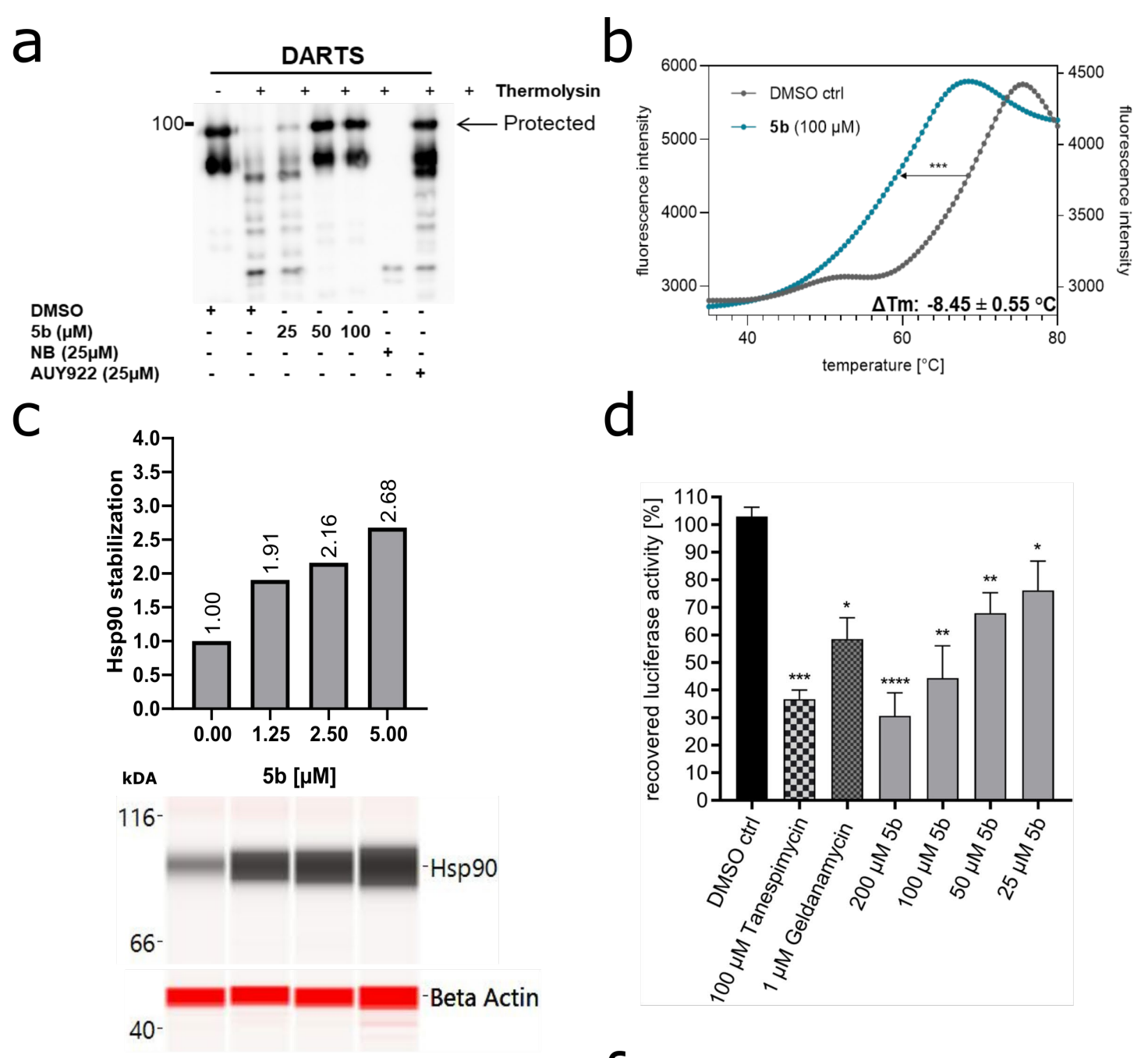

e
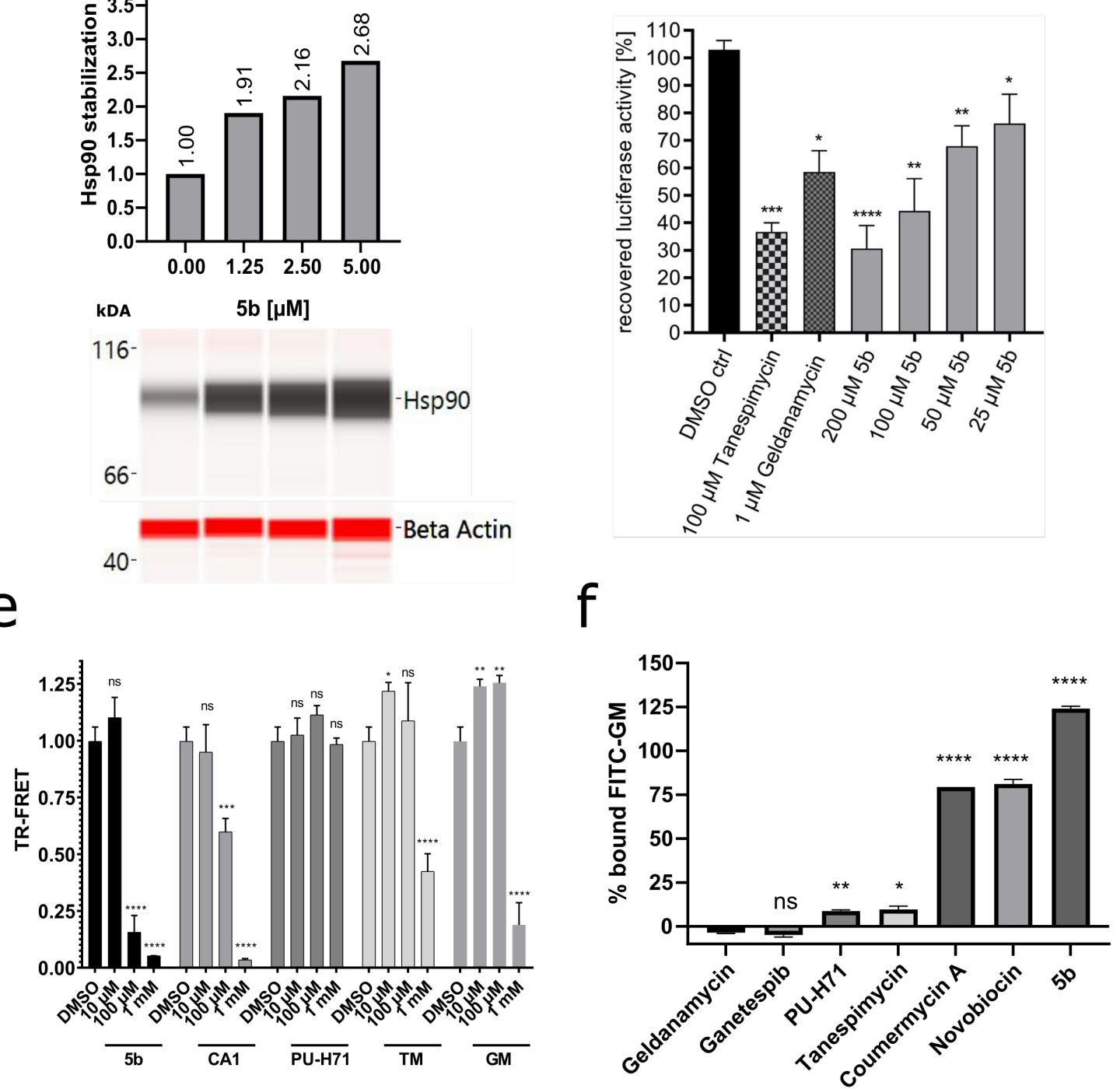

Figure 3: Specificity of 5b against Hsp90 CTD and its cochaperone function. a) Recombinant (full-length) Hsp90 $\alpha(1 \mu \mathrm{g})$ was incubated with $\mathbf{5} \mathbf{b}$ at indicated concentrations, followed by digestion with thermolysin. Treated protein samples were electrophoresed (SDS-PAGE) and immunoblotted with anti-Hsp90 $\alpha$ for detecting protection of $\mathrm{Hsp} 90 \alpha$ protein by $\mathbf{5 b}$ (the upper band is protected from proteolysis). b) Cell-free thermal shift assay was performed by incubating recombinant Hsp90 $\alpha$ CTD protein with $\mathbf{5 b}$, at an increasing temperature (up to $95{ }^{\circ} \mathrm{C}$ ). Melting temperature $\left(T_{m}\right)$ without inhibitors (DMSO) was used as a control. c) The dose-dependent intracellular (K562 cells) thermal stabilization (CETSA ITDRF) of Hsp90 after $\mathbf{5 b}$ incubation (24 h) at its increasing concentration $(1.25 \mu \mathrm{M}-5 \mu \mathrm{M})$. d) $\mathbf{5 b}$ inhibits Hsp90 $\alpha$ chaperone function, comparable to TM and GM, in cell-free luciferase refolding assay, where the incubation of the inhibitors prevented the rabbit reticulocyte lysate (a source of Hsp90) assisted refolding of denatured luciferase. e) Incubation of $\mathbf{5 b}$ blocked the binding of Hsp90 CTD-interacting 
cochaperone (PPID) in TR-FRET measurements. f) $5 \mathbf{b}$ did not reduce the amount of Hsp90-bound FITC-labelled GM and, therefore, does not compete for the GM binding pocket of full-length Hsp90 $\alpha$. Unlabelled GM, GP, PUH71, and TM served as positive controls and NB and CA1 as negative controls.

\section{Binding mode prediction of $\mathbf{5 b}$ at $H s p 90 \alpha$}

To provide structural insights how $\mathbf{5 b}$ binds to the CTD of human Hsp90, we performed 40 independent molecular dynamics (MD) simulations of free diffusion of $\mathbf{5 b}$ in the presence of truncated monomeric Hsp90 $\alpha$ (aa 294-699), using the Amber 18 suite of molecular simulation programs ${ }^{46}$ and the ff $14 \mathrm{SB}^{47}$ and a modified $\mathrm{GAFF}^{30,48}$ force field for protein and ligand. Initially, we generated 40 individual starting configurations by randomly placing $\mathbf{5 b}$ and the CTD structure, leaving at least $10 \AA$ between atoms in 5b and the CTD structure. After minimization, thermalization, and density adaptation, we performed MD simulations of $500 \mathrm{~ns}$ length, in which the $\mathbf{5 b}$ molecule diffused freely. To counter the high flexibility of the C-terminal helix interface, we introduced positional restraints on the backbone atoms, adjusting the reference coordinates every $100 \mathrm{~ns}$ to allow for moderate protein movements.

From the trajectories, first, we extracted all frames where $\mathbf{5 b}$ is bound to Hsp90 (no-fit RMSD of $\mathbf{5 b} \leq$ $1.5 \AA$ to the previous frame after superimposing Hsp90). Mapping the probability density of occurrence of $\mathbf{5 b}$ onto the surface of Hsp90 (Figure 4a) revealed two main binding regions: one in the C-terminal helix interface (Figure 4a, green), where binding occurred in 10 out of the 40 replicas, and another in a cleft between the CTD and middle domain (Figure 4a, blue), where binding occurred in 6 out of 40 replicas. In the latter case, an area of high density with the shape of $\mathbf{5 b}$ is observed (Figure 4a, light blue), which resulted from a single trajectory. This indicates that the ligand was kinetically trapped in this one case, although the position is thermodynamically not favorable. By contrast, the densities in the C-terminal interface are more ambiguously shaped, indicating that, while binding there is favorable, the ligand can still explore multiple binding modes, which are also seen to interchange.

To further study these binding modes, we clustered the bound frames of $\mathbf{5 b}$ mapped on the protein surface with respect to their RMSD after superimposing Hsp90. Among the binding modes were several that form interactions to the C-terminal helix interface, with $\mathbf{5 b}$ positioned such that it mimics interactions formed by H5' in the dimer (Figure 4c and d). To corroborate that this binding mode is favorable, we computed the effective binding free energies by the MM-GB/SA approach for the trajectory that led to it. Already first transient interactions with the protein resulted in effective energies 
down to $\sim-30 \mathrm{kcal} \mathrm{mol}^{-1}$. The effective energies decreased further to $\sim-45 \mathrm{kcal} \mathrm{mol}^{-1}$ once the ligand bound in the C-terminal interface, thereby forming interactions with Hsp90 that remained stable even when the trajectory was extended to $1 \mu \mathrm{s}$, indicating that such poses are particularly favorable (Figure 4b). As to the magnitude of the effective energies, note that configurational entropy contributions were not considered, since estimating such contributions by normal mode analysis may introduce additional uncertainties. $^{49,50}$

Overall, the probability density of bound $\mathbf{5 b}$ poses, the proportion of replicas, and the results of the MMGB/SA computations indicate that $\mathbf{5 b}$ preferentially binds to the C-terminal helix interface, where it can adopt poses that mimic $\mathrm{H} 5$ '.

\section{Comparison to $\mathbf{5 b}$ binding at $H s p 90 \beta$}

We then set out to study if there is an isoform specificity for the binding of $\mathbf{5 b}$ because the helical interface regions differ in three positions: $\alpha$ : S641, $\beta$ : P633; $\alpha$ : S658, $\beta$ : A650; $\alpha$ : A685, $\beta$ : S677 (SI Figure 21). Using the same setup as before, we performed MD simulations of free ligand diffusion around Hsp90ß. The probability density of bound $\mathbf{5 b}$ again revealed that the C-terminal helix interface is the most preferred region, followed by the cleft between the CTD and middle domain (SI Figure 24). Notably, no high density in this cleft was found now, in contrast to Hsp90 $\alpha$, confirming that the observation there resulted from kinetic trapping. Hence, despite the few sequence variations in the Cterminal helix interface between Hsp90 $\alpha$ and $\mathrm{Hsp} 90 \beta$, the same preferred binding region of $\mathbf{5 b}$ was found in both cases. 

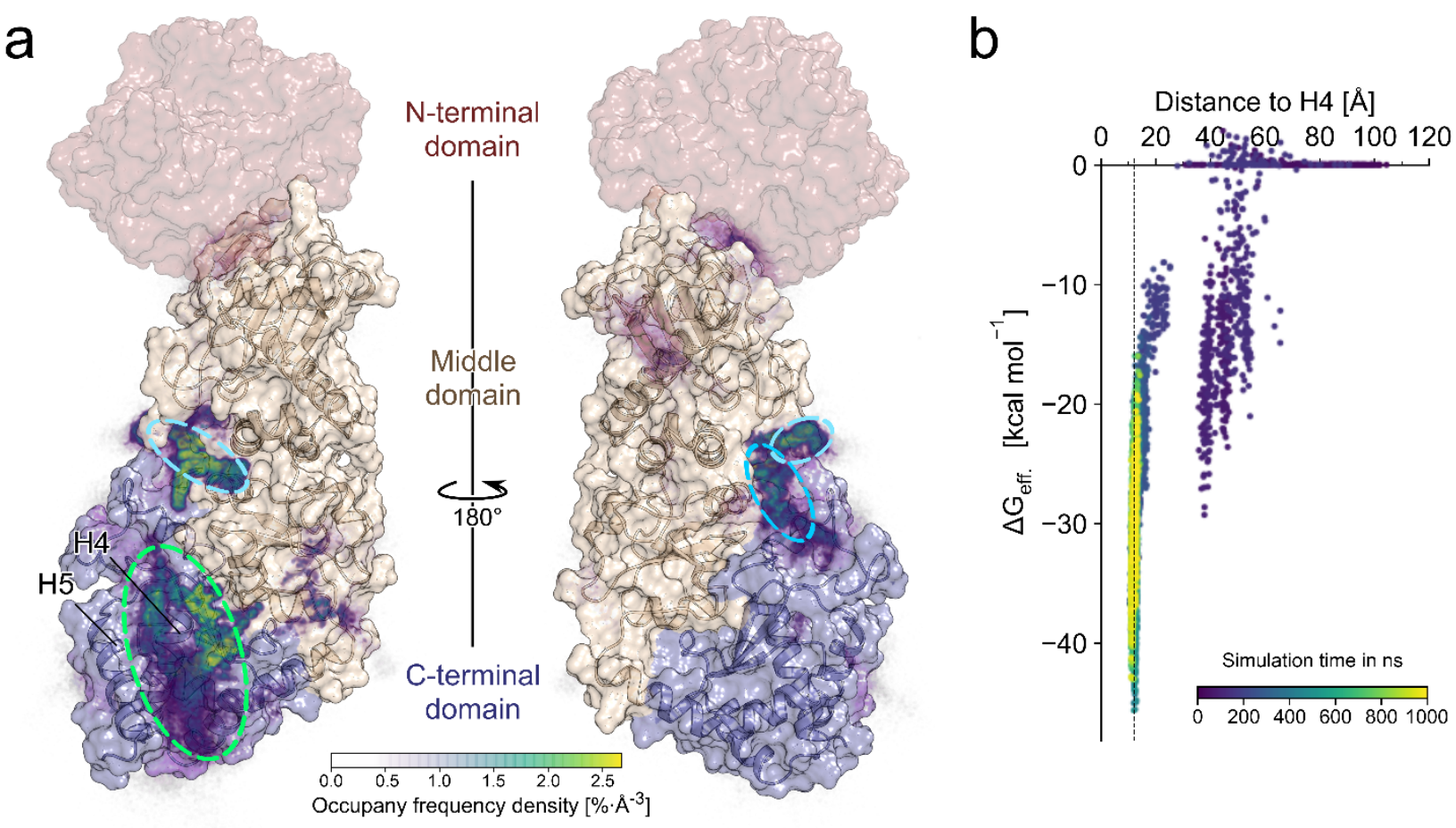

\section{C}
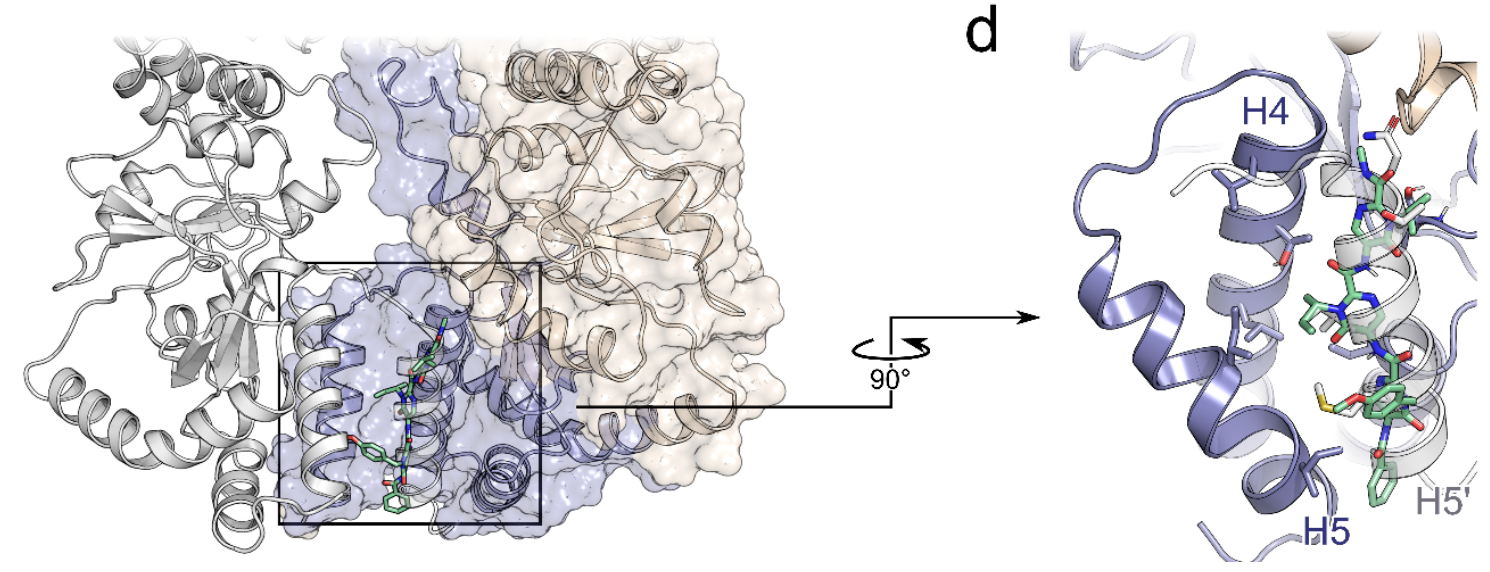

Figure 4: MD simulations of 5b diffusion and effective binding energy calculations to predict the binding mode.

a) The relative densities of the bound poses of $\mathbf{5 b}$ after $500 \mathrm{~ns}$ are mapped on the Hsp90 $\alpha$ monomer fragment used in the simulations (PDB ID 3q6m). The missing NTD is shown in red, based on the Hsp90 $\beta$ structure (PDB ID 5 fwk). Particularly high densities are observed in the region between H4 and H5 (green circle). A second, less preferred site is in the cleft between the CTD and middle domain (blue circles). b) Effective binding energy calculations over a single trajectory that resulted in $\mathbf{5 b}$ binding in the C-terminal helix interface as a function of the center-of-mass distance between $\mathbf{5 b}$ and $\mathrm{H} 4$ and the simulation time (see color scale). The dashed line at $12.1 \AA$ corresponds to the H4-H5' distance in the crystal structure of PDB ID 3q6m. c) Possible binding mode of $5 \mathbf{b}$ in the helix interface, where $\mathbf{5 b}$ mimics H5'. d) Blow-up of the possible binding mode of $\mathbf{5 b}$ showing how its side chains mimic side chains of $\mathrm{H} 5$ '.

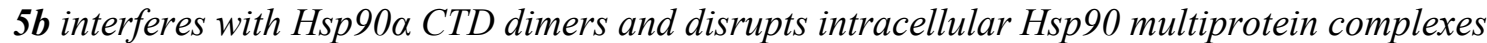

CTD dimerization of Hsp90 is necessary for its function. ${ }^{51,52}$ To study the effect of $\mathbf{5 b}$ exposure on the dissociation of Hsp90 dimers in a cell-free assay, we used Hsp90 $\alpha$ CTD protein after incubation with amine-reactive crosslinker $\mathrm{BS}^{3}$, as previously described. ${ }^{42,45}$ A dose-dependent reduction of Hsp90 $\alpha$ 
CTD dimers along with an increase in the high order oligomeric species was noticed upon incubation with 5b (Figure 5a). Next, we performed small-angle X-ray scattering (SAXS) with the Hsp90 $\alpha$ CTD protein, which was coupled to a size exclusion chromatography column (SEC-SAXS) at the ESRF beamline BM29 in Grenoble. ${ }^{53,54}$ In the absence of $\mathbf{5 b}$, a clear dimeric profile of the Hsp90 $\alpha$ CTD protein was visible on the chromatogram, with an additionally minor tetrameric species (SI Figure 25). We used the program CHROMIXS ${ }^{55}$ to merge the frames containing the dimer from this SEC-SAXS profile. Buffer frames were then subtracted using PRIMUS. ${ }^{56}$ From the SAXS data, a radius of gyration $(R g)$ of $3.23 \mathrm{~nm}$ was calculated, which describes the average particle dimension in solution. The ab initio model fit from DAMMIF ${ }^{57}$ shows a $\chi^{2}$ of 1.127 , indicating good agreement with the experimental data (Figure 5b and SI Table 2). The corresponding dimeric envelope is highlighted in Figure 5c, superimposed with the calculated dimeric model of Hsp90 $\alpha$ CTD. Further, we tested the effect of $\mathbf{5 b}$ on the Hsp90 CTD dimer using SAXS (Figure 5d). Due to the low solubility of $\mathbf{5 b}$, we needed to measure the sample as an ensemble of species in solution. First, we tested Hsp90 $\alpha$ CTD without $\mathbf{5 b}$ on Xeuss 2.0 with Q-Xoom system and observed an increase of $R g$ to $3.40 \mathrm{~nm}$. This increase is likely due to the small amount of tetramer in solution in the ensemble. Theoretically, the $R g$ of a tetrameric Hsp90 $\alpha$ CTD species is $3.78 \mathrm{~nm}$, using CRYSOL, indicating that even in batch mode SAXS measurements, the Hsp90 $\alpha$ CTD protein is predominantly in a dimeric state. We added $\mathbf{5 b}$ with an equimolar concentration to Hsp90 $\alpha$ CTD protein, and the $R g$ value slightly increased from $3.40 \mathrm{~nm}$ to $3.46 \mathrm{~nm}$. However, with an increasing concentration of $\mathbf{5 b}$ to $1 \mathrm{mM}$, the $R g$ value increased to $4.11 \mathrm{~nm}$. Compared to the theoretical $\mathrm{Rg}$ value of $3.78 \mathrm{~nm}$ for the tetramer, we observed that $\mathbf{5 b}$ induces oligomerization of Hsp90 $\alpha$ CTD to species even larger than the tetrameric form (Figure 5e). It is unclear, however, if the oligomers are formed from Hsp90 $\alpha$ CTD monomers or dimers, as the single species could not be resolved in the ensemble measurement.

In a cellular context, Hsp90 acts in multiprotein complexes. ${ }^{19}$ Therefore, interfering with Hsp90 function may lead to the disruption of these complexes. In a cellular assay, western blotting was performed under reducing (+dithiothreitol or + DTT) and non-reducing (-DTT) conditions after $5 \mathbf{b}$ incubation of the K562 cells. ${ }^{28}$ Similarly to $\mathrm{AX},{ }^{28} \mathbf{5 b}$ inhibited the formation of Hsp90 higher-order multimeric species, in contrast to novobiocin (NB), but in concordance with AUY922 (SI Figure 26). Next, to study the effect 
of $\mathbf{5 b}$ exposure on Hsp90 native multiprotein complexes, blue native (BN) PAGE analysis was carried out with K562 cell lysates after 5b incubation. ${ }^{19,28}$ At cytotoxic concentrations of $\mathbf{5 b}$, Hsp90a and Hsp90 $\beta$ multiprotein complexes were disrupted, including monomers/dimers of Hsp40 and Hsp27; Hsp60 (primarily in mitochondria) multiprotein complexes, serving as a loading control, were not affected (Figure 5e).

Taken together, these results confirm that $\mathbf{5 b}$ interferes with the Hsp90 $\alpha$ CTD dimerization, induces oligomerization, and disrupts intracellular Hsp90 multiprotein complexes.

\section{Basic physicochemical properties and microsomal stability of $\mathbf{5 b}$}

Next, we assessed the aqueous solubility, chemical stability, and in vitro metabolic stability of $\mathbf{5 b}$. The thermodynamic solubility of $\mathbf{5 b}$ was determined in phosphate-buffered saline (PBS, $25^{\circ} \mathrm{C}, \mathrm{pH} 7.4$ ) after 4 and 24 h incubation time (Supplementary Note 2). Ondansetron was used as a reference compound with high solubility of $95 \mu \mathrm{M}$. The thermodynamic solubility of $\mathbf{5 b}$ was low, ranging from $4 \mu \mathrm{M}$ after 4 $\mathrm{h}$ to $8 \mu \mathrm{M}$ after $24 \mathrm{~h}(\mathrm{n}=2)$.

To study the chemical stability of $\mathbf{5 b}$ at physiological $\mathbf{p H}$, the compound was dissolved in a mixture of Tween20/ethanol/phosphate buffer pH 7.5 (7/3/90) and monitored over 24 h (Supplementary Note 2). After $24 \mathrm{~h}$, almost no decomposition was detected $(0.7 \%$ drug decomposition, $\mathrm{n}=2)$. The chemical stability of $\mathbf{5 b}$ at acidic $\mathrm{pH}$ was determined by dissolving $\mathbf{5 b}$ in a mixture of Tween20/ethanol/phosphate buffer pH 2 (7/3/90) and monitoring over $24 \mathrm{~h}$. After $24 \mathrm{~h}$, only slight decomposition was detected ( $1.3 \%$ drug decomposition, $\mathrm{n}=2$ ).

Metabolic stability screening of $\mathbf{5 b}$ in human liver microsomes revealed $91 \%$ stability after a 40 min incubation at $37 \mathrm{C}$ (Supplementary Note 2). Propanolol, a reference drug with medium to high metabolic stability, showed $74 \%$ of the parent compound remaining, and therefore demonstrated slightly decreased stability compared with $\mathbf{5 b} .{ }^{13}$ Calculated results for the intrinsic clearance suggest that $\mathbf{5 b}(6 \mu \mathrm{L} / \mathrm{min} / \mathrm{mg})$ is a low-clearance compound with estimated long half-life $(\mathrm{n}=2)$. 
a

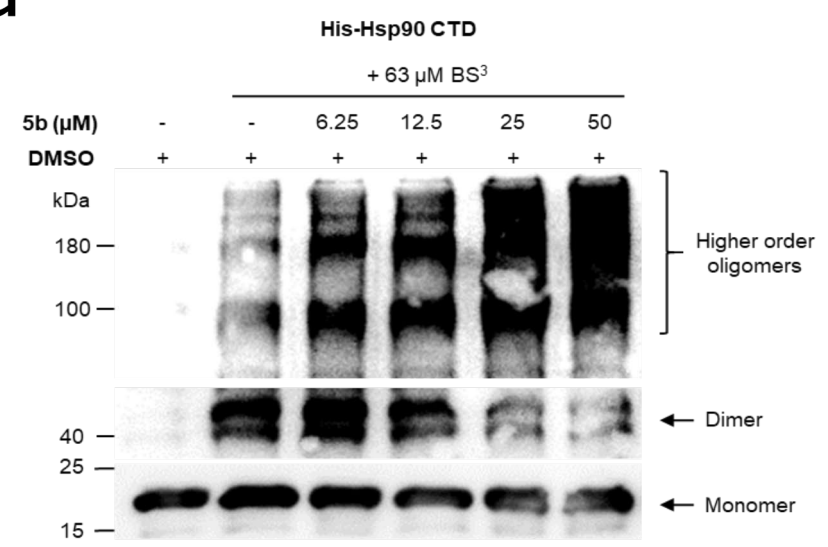

C

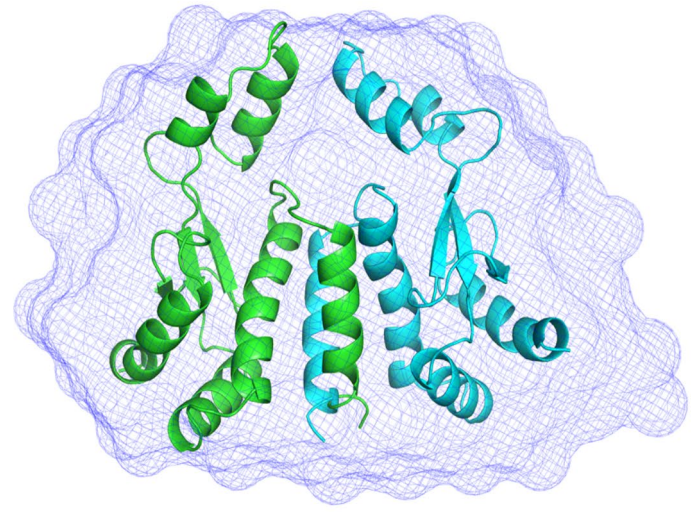

d

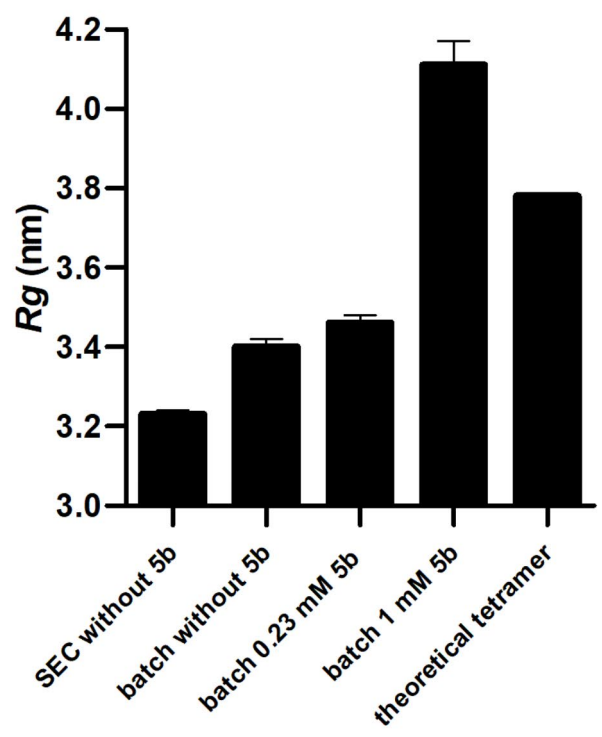

b

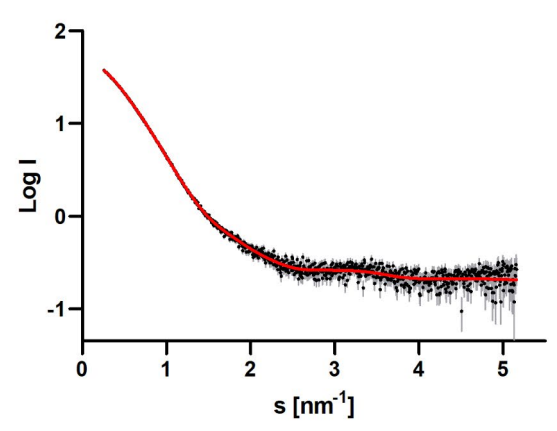

n

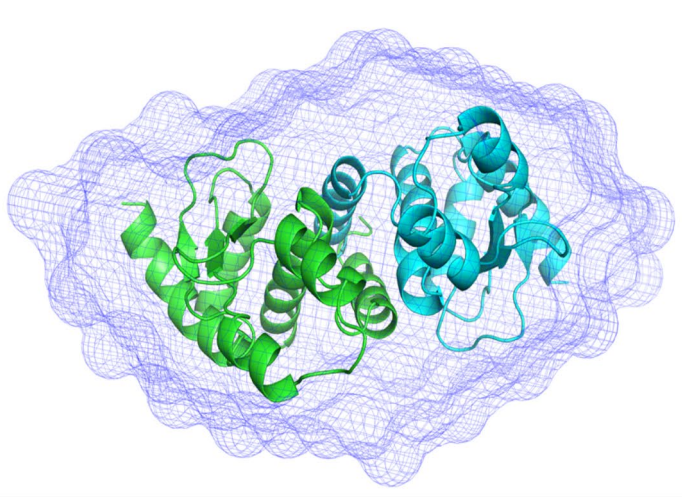

e

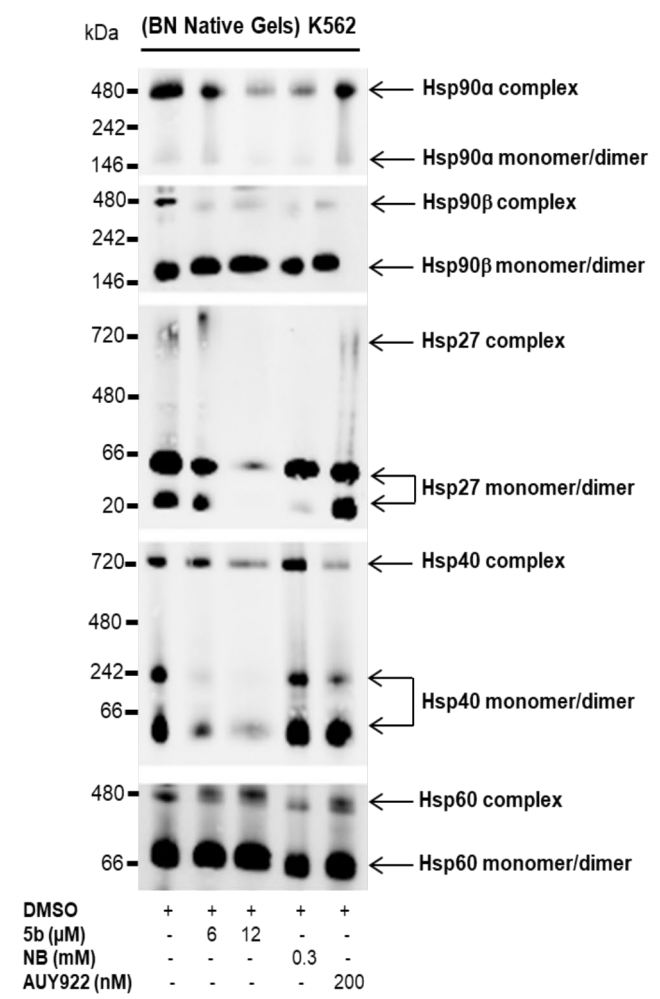


Figure 5: Effect of 5b on Hsp90 oligomeric species and CTD-mediated dimerization. a) Recombinant Hsp90 $\alpha$ CTD was incubated with $63 \mu \mathrm{M} \mathrm{BS}^{3}$ crosslinker, with (at indicated concentration) or without $\mathbf{5 b}$, followed by immunoblotting with anti-Hsp90 (AC88) antibody. b) Scattering data of Hsp90 $\alpha$ CTD is shown in black dots, with grey error bars. The ab-initio DAMMIF model fit is shown as a red line. The intensity is displayed as a function of momentum transfer s. c) The volumetric envelope, calculated from the scattering data using DAMMIF ${ }^{57}$, is shown as a blue surface. The monomers of the predicted Hsp90 CTD dimer model are shown in green and cyan. Superimposing was done using SUPCOMB.$\left.^{57} \mathbf{d}\right)$ The Radius of gyration $(R g)$ of the different Hsp90 $\alpha$ CTD protein samples was calculated using the Guinier approximation. ${ }^{58}$ The theoretical $R g$ of the tetramer was calculated using CRYSOL based on the structure PDB ID $3 \mathrm{q} 6 \mathrm{~m} .{ }^{59}$ e) Native Hsp90 complexes in K-562 (24h administration of 5b) was identified by running Blue Native (BN) gels followed by immunoblotting analysis. The cytotoxic concentration of $\mathbf{5 b}$ resulted in the potent disruption of Hsp90a, Hsp90ß, Hsp40, and Hsp27 complexes and monomers/dimers. AUY922 exposure elevated the expression of HSR associated protein complexes and monomers/dimers (Hsp40 and Hsp27), whereas Hsp60 served as loading controls.

\section{$\mathbf{5 b}$ is effective against resistant leukemia cells and in the zebrafish xenotransplantation model}

Elevated Hsp90 expression is reported in several resistant leukemia subtypes such as BCR-ABL1 ${ }^{+}$ CML/BCP-ALL, FLT3-ITD-driven AML, and Ph-like BCP-ALL. ${ }^{60-63}$ Besides that, client proteins of Hsp90 include several kinases (e.g., AKT, BCR-ABL1, BRAF, EGFR2, HER2, and JAK1/2), growth and steroid receptors, and apoptotic factors (e.g., BCL-2 and mutant p53), which are often aberrantly regulated in several malignancies. ${ }^{2,64}$ Therefore, we determined the efficacy of $\mathbf{5 b}$ on therapy-resistant cell lines obtained from different leukemia subtypes (B-ALL, T-ALL, CML, and AML), including imatinib (IM), multi TKI-resistant and bortezomib (BTZ)-resistant leukemic cell lines. ${ }^{28}$ Average $\mathrm{IC}_{50}$ values of $\mathbf{5 b}$ in these cell lines were determined using ATP-based viability assay and plotted in a clustered heat map, indicating the superior efficacy against BCR-ABL1+ and AML leukemic cell lines, when compared to T-ALL cell lines (Figure 6a).

As previously performed with $\mathrm{AX}^{28}$, we next determined the destabilizing effect of $\mathbf{5 b}$ on $\mathrm{BCR}-\mathrm{ABL} 1$ expression and associated downstream signaling. In K562 cells, 48 h exposure of $\mathbf{5 b}$ down-regulated the phospho-BCR-ABL1 and total-BCR-ABL1 levels, as well as the related downstream signaling pathways, as evaluated by immunoblot analysis (Figure $6 \mathbf{b}$ ). $\mathbf{5 b}$ additionally reduced the expression of client proteins associated with Hsp90 chaperone activity, involving Akt, Stat5, and c-Myc (Figure 6b). In contrast to AUY922, the exposure of $\mathbf{5 b}$ on the K562 cells did not induce the expression of Hsp70, Hsp40, and Hsp27 involved in HSR (Figure 6c). Exposure of 5b to the leukemic cell lines (K562, KCL22 and HL60) inhibited their proliferation (SI Figure 27) and induced apoptosis in a caspase 3/7 enzyme-dependent assay, with induction of approximately two- to eight-fold increase of apoptotic cells, in accordance to the reference Hsp90 CTD-targeting inhibitor, novobiocin (NB) (SI Figure 28). 
Besides, $\mathbf{5 b}$ facilitated early differentiation measured by expression of differentiation markers involving CD14 vs. CD11b in HL60 cells, and CD133 vs. CD11b in Mutz-2 cells (SI Figure 29). In this line, $48 \mathrm{~h}$ exposure of $\mathbf{5 b}$ to K562 cells significantly reduced the colony-forming capacity (SI Figure 30). To further evaluate the efficacy of $\mathbf{5 b}$ on leukemic cells, we used the zebrafish xenotransplantation model ${ }^{65}$ (Figure 6d). MOLT-4 cells were transplanted into zebrafish embryos at 32 hours post-fertilization (hpf). At this stage, the adaptive immune system is not yet developed, therefore, human cells can be tolerated by the host. The transparency of zebrafish embryos also enabled us to monitor the distribution of human cells, which were stained with a vital fluorescent dye. Xenotransplanted embryos were treated with AUY922 $(500 \mathrm{nM})$ and $\mathbf{5 b}(500 \mathrm{nM})$ for $48 \mathrm{~h}$, whereas DMSO was used as a negative control. No toxicity of drugs at the given concentration was noticed on the development of xenotransplanted embryos. We then determined the number of MOLT4 cells in each treated group using flow cytometry. Compared to the DMSO-treated group, the number of transplanted cells was significantly reduced in AUY922- and 5b-treated embryos.

To conclude, this data confirms the anti-leukemic effect of $\mathbf{5 b}$ without inducing HSR. 


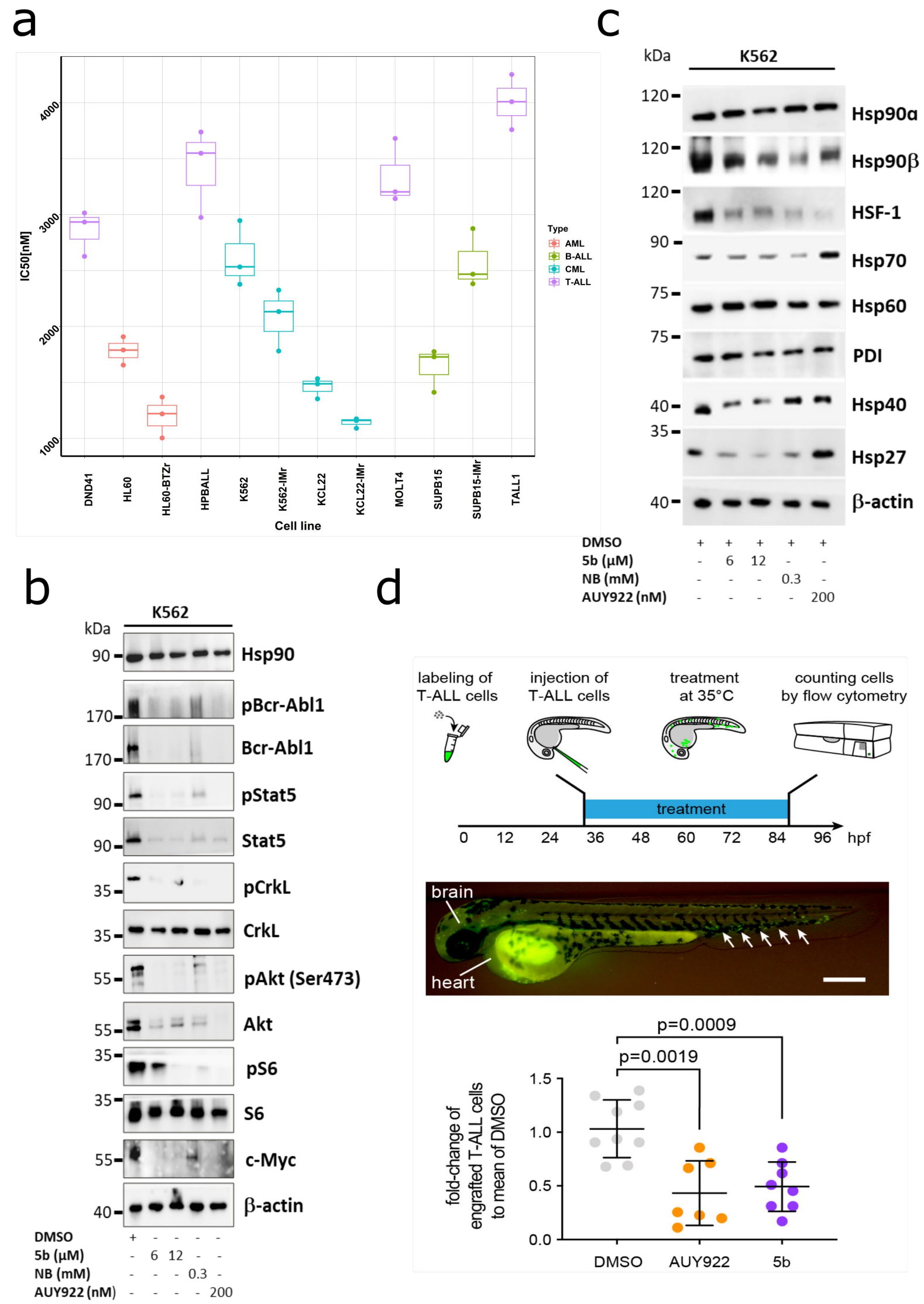

Figure 6: $5 \mathrm{~b}$ is effective against human leukemic cell lines without inducing any HSR. a) Comparative cytotoxicity assessment of different sub-groups of leukemic cell lines (K562, KCL22, SUPB15, HL60, MOLT4, DND41, TALL1 and HPBALL), imatinib-resistant cell lines (K562-IMr, KCL22-IMr, SUPB15-IMr), and bortezomib-resistant cell line (HL60-BTZr), after $72 \mathrm{~h}$ exposure to $\mathbf{5 b}$. The $\mathrm{IC}_{50}$ data was plotted as a clustered 
heat map, followed by unsupervised hierarchical clustering. The vertical axis of the dendrogram exemplifies the dissimilarity between clusters, whereas the color of the individual cell is related to its position along a $\log \mathrm{IC}_{50}$ $(\mu \mathrm{M})$ gradient. b) Treatment of K562 cells with $\mathbf{5 b}$ and respective controls (AUY922 and NB) for $48 \mathrm{~h}$ resulted in the downregulation of BCR-ABL1+ and subsequently downstream signaling pathways including phosphorylated and unphosphorylated Stat5a, Crkl, Akt, S6 (mTOR), and cMyc. c) K562 cells were treated with the indicated (cytotoxic) concentration of $\mathbf{5 b}, \mathrm{NB}$, and AUY 922 for $48 \mathrm{~h}$, and later protein lysates were subjected to immunoblot analysis. As expected, 5b and NB did not induce expression of Hsp70, Hsp40, and Hsp27, whereas AUY922 leads to HSR induction. Hsp60 (primarily present in mitochondria) and PDI (endoplasmic reticulum) served as a control. d) Upper panel describes the experimental rationale. (Middle panel) A representative image of xenotransplanted zebrafish embryo at $32 \mathrm{hpf}$. Scale bar, $250 \mu \mathrm{m}$. Note human T-ALL cells (green) were distributed in the yolk, brain, and hematopoietic tissue (arrows). (Lower panel) Fold-change of labeled cells normalized to the average percentage of labeled cells in the DMSO-treated group. Each dot represents three embryos pooled as one biological sample. Data are mean \pm standard deviation. The $p$-values were calculated with the Mann-Whitney test.

\section{$5 b$ acts on TKI-resistant BCR-ABL1+ leukemic cells}

The integration of specific tyrosine kinase inhibitors (TKI) such as imatinib (Gleevec) into polychemotherapy treatment protocols has significantly improved the response rate in BCR-ABL1+ leukemia patients (initial remission went from $35 \%$ to $88 \%$ ). ${ }^{66}$ However, stable remission cannot be sustained in many cases as the leukemic cells pursue several escape mechanisms against TKI treatment; one of them is the occurrence of mutations in the ABL1 kinase domain. Especially, in the BCR$\mathrm{ABL1}^{\mathrm{T} 315 \mathrm{I}}$ mutant, only ponatinib (TKI) is effective ${ }^{67}$, albeit with severe cardiovascular side effects. ${ }^{68}$ As Hsp90 facilitates the correct folding of several oncogenic newly synthesized or denatured proteins, among them BCR-ABL1, ${ }^{69-71}$ therefore targeting Hsp90 with small molecule inhibitors would destabilize BCR-ABL1 and can serve as a therapeutic target. ${ }^{3,10} \mathrm{We}$, therefore, next tested the efficiency of $\mathbf{5 b}$ in a murine BA/F3 cell line stably expressing clinically relevant mutants (BCR-ABL1 ${ }^{\mathrm{T} 315 \mathrm{I}}$, BCR$\mathrm{ABL1}^{\mathrm{E} 255 \mathrm{~K}}$, and BCR-ABL1 ${ }^{\mathrm{M} 351 \mathrm{~T}}$ ) with prominent TKI resistance profiles. ${ }^{28}$ As expected, similar to K562 cells, exposure of $\mathbf{5 b}$ significantly reduced proliferation (Figure 7a) and induced apoptosis (Figure 7b) at 6 and $12 \mu \mathrm{M}$, comparable to NB (at $0.3 \mathrm{mM}$ ) in BA/F3 cells expressing BCR-ABL1 ${ }^{\mathrm{T} 315 \mathrm{I} \text {, }}$ E255K, or M351T mutants. Additionally, after the application of $\mathbf{5 b}$, BCR-ABL1 oncoprotein was destabilized, and downstream signaling pathways (Akt and Stat5) were blocked with increasing concentrations of $\mathbf{5 b}$, comparable to the human leukemic cell lines (Figure 7c). Furthermore, $24 \mathrm{~h}$ exposure of $\mathbf{5 b}$ on BCR-ABL1 ${ }^{\mathrm{T} 315 \mathrm{I}, \mathrm{E} 255 \mathrm{~K} \text { or M351T }}$ mutants-expressing BA/F3 cells significantly inhibited the colony formation ability (Figure 7d). In addition, in our generated human BCR-ABL1+ IM-resistant cell line models (K562-IMr and KCL22-IMr), ${ }^{28} \mathbf{5 b}$ did not differentiate in inducing apoptosis between IM-resistant vs. IM-sensitive clones, proposing a superior effect of $\mathbf{5 b}$ in human IM-resistant BCR- 
ABL1-positive cells (Figure 7e and SI Figure 31). Encouraged by these results, we next tested 5b on three primary $\mathrm{CML}^{\mathrm{CD} 34+} \mathrm{IM}$-resistant patient samples in the range of cytotoxic concentration $(5-10 \mu \mathrm{M})$ obtained from leukemic cell lines. Similar to BCR-ABL1+ leukemic cell lines, the exposure of $\mathbf{5 b}$ reduced the proliferation, induced apoptosis, and reduced the colony-forming ability of $\mathrm{CML}^{\mathrm{CD} 34+} \mathrm{IM}-$ resistant patient cells and also revealed a potent combinatorial inhibitory effect when used in combination with IM (Figure 7f $-\mathbf{7 h})$. 
a

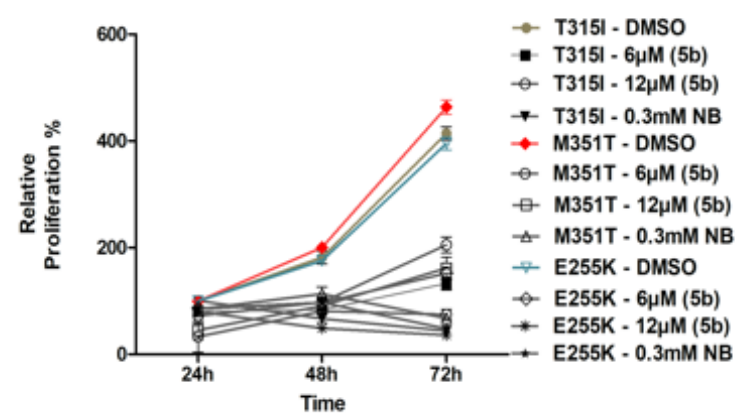

C

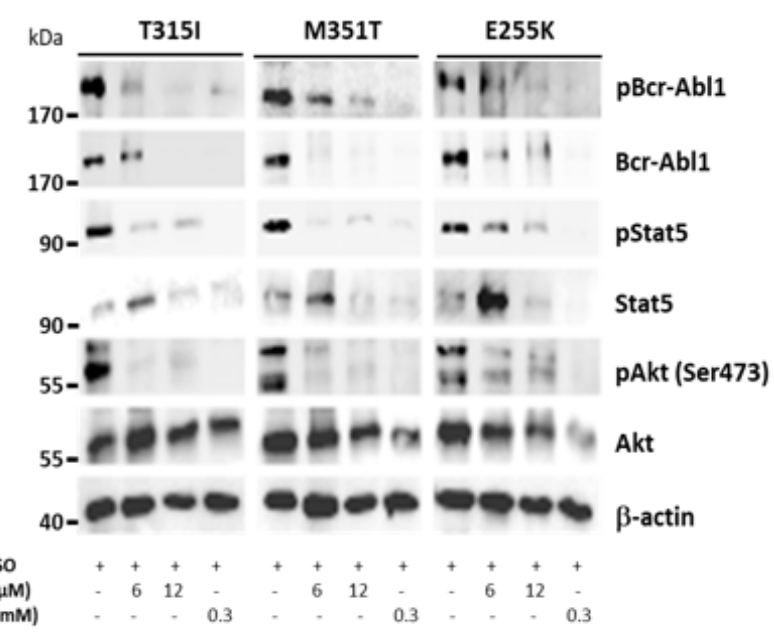

e

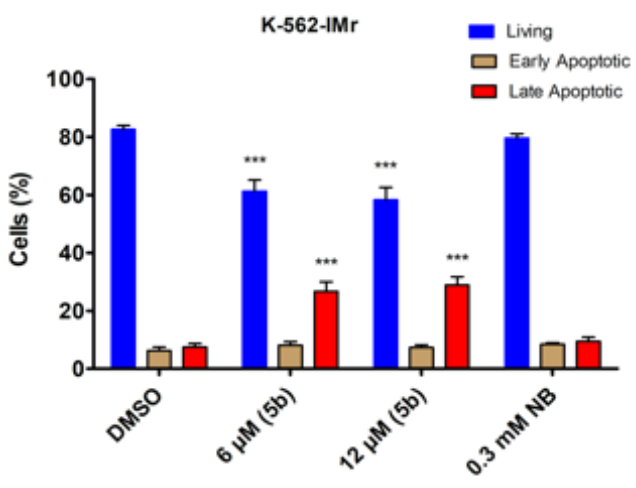

g

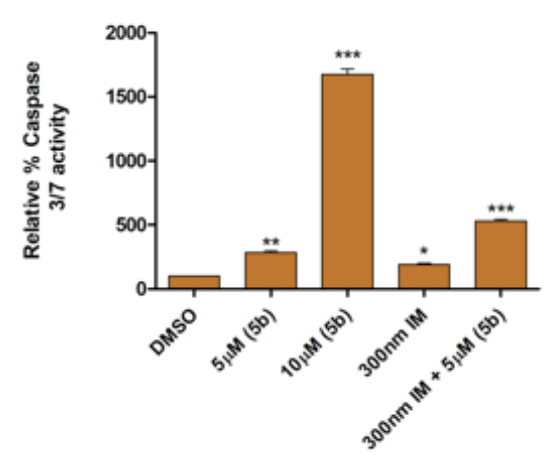

$\mathrm{h}$ b

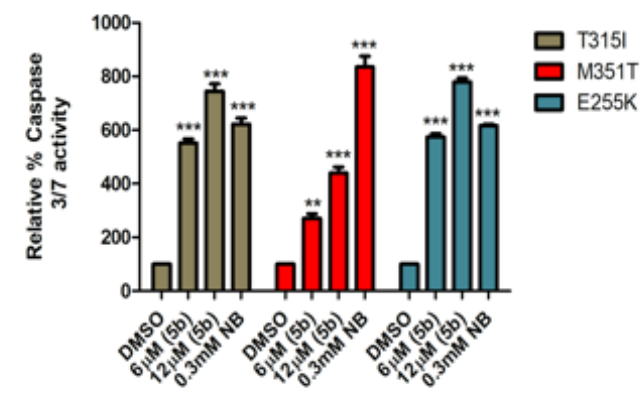

d

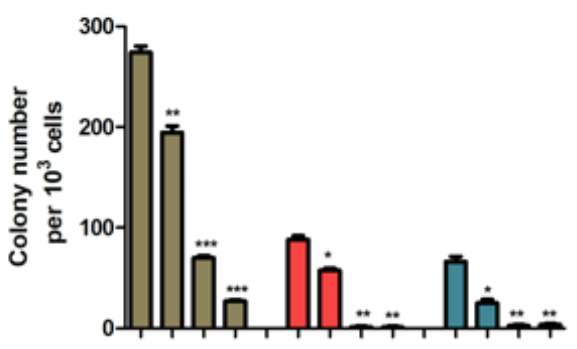

క5
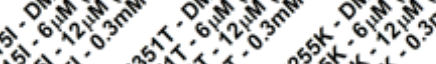

f
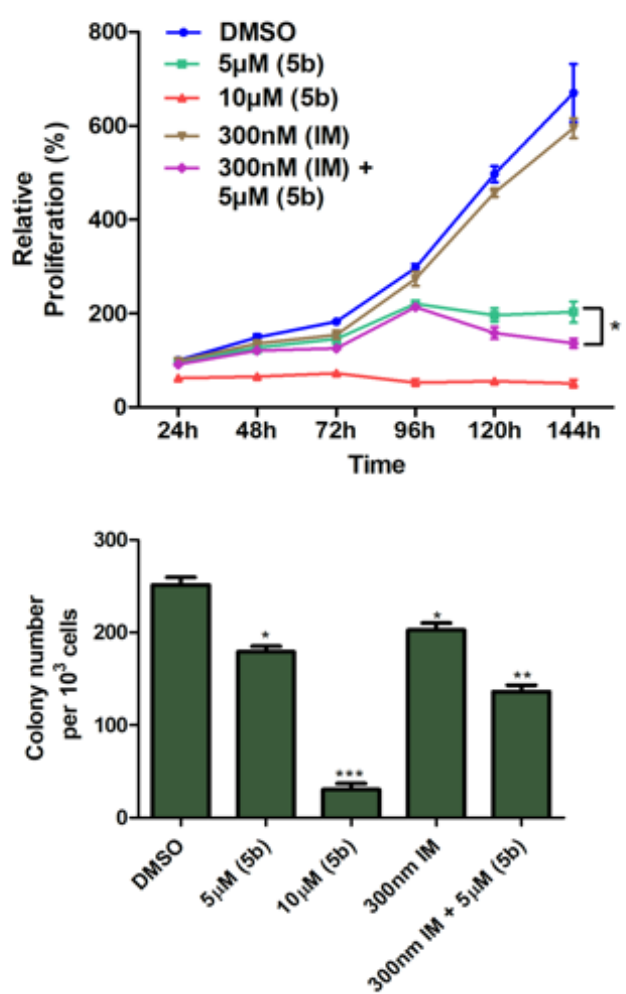

Figure 7: 5b as a potent inhibitor against TKI-resistant BCR-ABL1 mutant and a primary patient sample. a) BA/F3 cells expressing BCR-ABL1 mutants (T315I, M351T \& E255K) were treated with the indicated concentration of $\mathbf{5 b}$, and later the viable cells were counted after every $24 \mathrm{~h}$ interval for 3 days. b) Apoptosis induction of the same cells after $48 \mathrm{~h}$ incubation of the compounds at indicated concentration, determined by Caspase 3/7 dependent Glo assay. c) Exposure of $\mathbf{5 b}$ to these cells destabilizes BCR-ABL1 and subsequently its 
associated downstream signaling pathways. d) BA/F3 BCR-ABL1 mutant-expressing cells were seeded in methylcellulose-based semisolid medium, after $24 \mathrm{~h}$ treatment with $\mathbf{5 b}$. Colonies were counted after 14 days. e) $\mathrm{K} 562-\mathrm{IMr}$ cells were treated with the indicated concentration of $\mathbf{5 b}$ for $48 \mathrm{~h}$, later dually stained with annexin V/PI, and subsequently measured by FACS. f) Primary $\mathrm{CML}^{\mathrm{CD} 34+}$ patient cells were treated with the indicated concentration of $\mathbf{5 b}$ or IM alone or in combination, and later the viable cells were counted after every $24 \mathrm{~h}$ interval for 6 days. g) Apoptosis induction in primary $\mathrm{CML}^{\mathrm{CD} 34+}$ patient cells after exposure of $\mathbf{5 b}$ or IB alone or in combination. g) Primary $\mathrm{CML}^{\mathrm{CD} 34+}$ patient cells were seeded in methylcellulose medium after $24 \mathrm{~h}$ treatment with $\mathbf{5 b}$, IM alone or both in combination. Colonies were counted after 14 days. Columns depict the mean of three independent experiments $(n=3)$.

\section{Discussion}

The CTD of Hsp90 contains several binding areas: the C-terminal ATP binding site, the MEEVD motif at the end of the CTD, and the primary dimerization interface of $\mathrm{Hsp} 90 .{ }^{23}$ The C-terminal ATP binding site is only available upon occupation of the N-terminal ATP binding pocket and binds purine and pyrimidine nucleotides, while the N-terminal ATP binding site (NTD) is more specific for adenine derivatives. $^{72}$ The MEEVD motif binds the TRP-domain of cochaperones such as Hop and immunophilins, which is formed by a four-helix bundle and is crucial for Hsp90 dimerization. ${ }^{2}$ Proteinprotein interactions in the interface of the four-helix bundle maintain the dimeric state of Hsp90. ${ }^{51}$

In this study, we present the development of the first-in-class small-molecule inhibitor of Hsp90, $\mathbf{5 b}$, which was rationally designed to target the C-terminal dimerization interface. Based on our prior hotspot prediction ${ }^{26}$ and the prototype compound $\mathrm{AX}^{28}$, we successfully performed a scaffold-hopping from the aminoxy peptide-backbone towards more drug-like tripyrimidones. $\mathbf{5 b}$ mimics $\alpha$-helical side-chains that form hotspot residues located on H5 in the dimerization interface. To independently predict the binding site and mode of $\mathbf{5 b}$, we performed extensive MD simulations, in which the inhibitor was allowed to diffuse freely around an MD-CTD construct of Hsp90a, similar to our analysis on AX binding ${ }^{28}$ and related studies. ${ }^{73-75}$ The results revealed the C-terminal dimerization interface as the most likely binding region of $\mathbf{5 b}$, which was confirmed by effective binding energy computations, corroborating the hypothesis underlying the design of $\mathbf{5 b}$. Following the high sequence-identity in the interface region, similar results were obtained for Hsp90 $\beta$, suggesting that $\mathbf{5 b}$ does not exhibit isoform specificity.

Next, the selective binding of $\mathbf{5 b}$ to Hsp90 was validated in a variety of biochemical and cellular assays, including DARTS, thermal and isothermal shift assays, whereas intracellular Hsp90 engagement and 


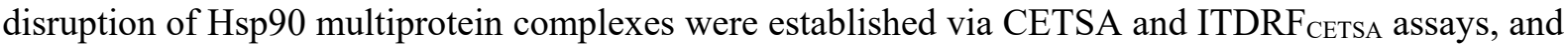
immunoblotting under reducing conditions (+/- DTT) and non-denaturing (BN-PAGE) conditions. Moreover, a crosslinker and auto display dimerization assay, as well as SEC-SAXS measurements, repeatedly confirmed the destabilization of Hsp90 $\alpha$ CTD dimers upon $\mathbf{5 b}$ incubation, whereas no unspecific binding of $\mathbf{5 b}$ was reported on the Hsp90 $\alpha$ NTD protein in an FP-based competitive assay. However, during SAXS measurements, which were performed on an ensemble of species in solution, we were unable to determine whether $\mathbf{5 b}$-induced oligomers originated from Hsp90a CTD monomeric or dimeric species. In addition, 5b blocks the chaperone function of Hsp90, as determined by TR-FRET assay and in cell-free luciferase refolding assay. Markedly, even though there are differences in the inhibitory concentrations against tested leukemia cells between $\mathbf{5 b}\left(\mathrm{IC}_{50}\right.$ in submicromolar range) and reference Hsp90 NTD control inhibitors ( $\mathrm{IC}_{50}$ in subnanomolar range), a comparative selectivity profile (in cell-free or cell-based biochemical assays) toward Hsp90 was observed between $\mathbf{5 b}$ and Hsp90 NTD reference inhibitors. This data indicate that the conventional Hsp90 NTD inhibitors induce cellular inhibitory effects through their off-target activity, besides targeting Hsp90. ${ }^{4,16}$

Moreover, $\mathbf{5 b}$ exhibited potent in vitro anticancer activity against a broad spectrum of therapy-resistant leukemia cell lines (including TKI and proteasome inhibitor-resistant) and primary TKI-resistant (BCRABL1+) leukemia patient cells. 5b significantly reduced the leukemia burden in the zebrafish xenotransplantation model and induced apoptosis in TKI-resistant BCR-ABL1 ${ }^{\mathrm{T} 315 \mathrm{I} \text {, E255K or M351T }}$ mutant cells by destabilizing the BCR-ABL1 expression and, thereby, hampering related downstream signaling cascades without HSR induction. These data collectively established $\mathbf{5 b}$ as a first-in-class smallmolecule inhibitor that targets the C-terminal dimerization interface.

\section{Conclusion}

Through structure-based molecular design, chemical synthesis, molecular simulations-based prediction of the binding mode, and evaluation of biochemical affinity, we have developed the first low-molecular weight compound interfering with the Hsp90 CTD dimerization. The C-terminal Hsp90 inhibitor 5b contains a tripyrimidonamide scaffold and is active against therapy-resistant leukemia cells as well as 
in a zebrafish xenotransplantation model without exhibiting the pro-survival resistance mechanism HSR.

\section{Methods}

Chemical synthesis: See Supplementary Note 1 for general methods, synthetic protocols, compound characterization, and spectral data (SI Figure 1 - 20).

Expression and purification of recombinant Hsp90: Human Hsp90 $\alpha$ CTD (563-732 amino acids) recombinant protein purification was performed as previously described.$^{26}$ Human Hsp90 $\alpha$ NTD (amino acids 9-236; Addgene \#22481) protein was expressed in E. coli BL21-DE3 cells. ${ }^{76}$ BL21-DE3 expression strains were grown overnight and used to inoculate LB medium at $37^{\circ} \mathrm{C}$ supplemented with $100 \mu \mathrm{g} / \mathrm{mL}$ ampicillin to an $\left(\mathrm{OD}_{600}=0.5-0.8\right)$, followed by overnight induction of protein expression with $0.5 \mathrm{mM}$ Isopropyl $\beta$-D-1-thiogalactopyranoside (IPTG) at $25^{\circ} \mathrm{C}$. After induction, cells were harvested by centrifugation at $5000 \times \mathrm{g}$ and lysed using B-PER bacterial protein extraction reagent (ThermoFisher Scientific, Wesel, Germany). GST-tagged Hsp90 CTD and NTD proteins were affinity purified spin columns (ThermoFisher Scientific) and eluted using glutathione elution buffer. Protein aliquots were made and supplemented with $5 \%$ glycerol and stored at $-80{ }^{\circ} \mathrm{C}$.

Microscale thermophoresis (MST): The labeling of the CTD of Hsp90 (Hsp90 CTD) was described before. ${ }^{26,28}$ For determination of the apparent $K_{\mathrm{D}}$ value of Hsp90 CTD and the respective inhibitor, $10 \mu 1$ of $50 \mathrm{nM}$ labeled Hsp90 CTD in PBS containing $0.5 \%$ BSA and $0.1 \%$ Tween-20 were mixed with $10 \mu \mathrm{l}$ of the respective inhibitor in different concentrations ranging from $15 \mathrm{nM}$ to $500 \mu \mathrm{M}$ and incubated for $15 \mathrm{~min}$ at RT in the dark. Thermophoresis of each mixture was induced at $1475 \mathrm{~nm} \pm 15 \mathrm{~nm}$ and measured using the Monolith NT.115 (NanoTemper Technologies GmbH, München, Germany) ${ }^{77}$. The measurement was repeated three times independently for each sample and each measurement was performed at $25{ }^{\circ} \mathrm{C}$ for $25 \mathrm{~s}$ at $70 \%$ LED power and $40 \%$ infrared laser power. The resulting fluorescence values were plotted against the concentration of the titrant and the apparent $K_{\mathrm{D}}$ was calculated using the $K \mathrm{D}$ Fit formula $\mathrm{Y}=\mathrm{E}+(\mathrm{A}-\mathrm{E}) / 2 *\left(\mathrm{~T}+\mathrm{x}+K_{\mathrm{D}}-\operatorname{sqrt}\left(\left(\mathrm{T}+\mathrm{x}+K_{\mathrm{D}}\right)^{\wedge} 2-4 * \mathrm{~T} *\right.\right.$ 
x)) by GraphPad Prism software (GraphPad Software, Inc. La Jolla, USA). T, the concentration value for the labeled Hsp90 CTD, was set to $50 \mathrm{nM}$.

Autodisplay dimerization assay: $E$. coli strain $\mathrm{BL} 21(\mathrm{DE} 3)$ [B, $\mathrm{F}^{-}, d c m$, ompT, lon, hsdS ( $\left.\mathrm{rB}^{-} \mathrm{mB}^{-}\right)$, gal, $\lambda(\mathrm{DE} 3)]$ pETSH-3 Hsp90 encoding CtxB signal peptide, AIDA-I autotransporter and Hsp90 was used for the surface display of Hsp90. ${ }^{27,28}$ E. coli strain BL21 (DE3)[B, $\mathrm{F}^{-}, d c m$, ompT, lon, hsdS $\left(\mathrm{rB}^{-} \mathrm{mB}^{-}\right)$, gal, $\lambda(\mathrm{DE} 3)]$ pETSH-3 SDH08 encoding CtxB signal peptide, AIDA-I autotransporter and sorbitol dehydrogenase was used as control ${ }^{37}$. Recombinant bacteria were routinely grown at $37^{\circ} \mathrm{C}$ in lysogeny broth (LB) containing carbenicillin $(100 \mu \mathrm{g} / \mathrm{ml})$. Cells were grown to the mid log phase $\left(\mathrm{OD}_{600}=0.5\right)$ and the protein expression was induced by adding $1 \mathrm{mM}$ IPTG for $16 \mathrm{~h}$ at $30^{\circ} \mathrm{C}$. Cells were then washed three times and suspended in PBS to an $\mathrm{OD}_{600}=0.35$ in a final volume of $100 \mu 1$. Prior to p53-FITC incubation at a final concentration of $1 \mu \mathrm{M}$ for 1 hour at RT in the dark, cells were incubated for 15 minutes either with or without inhibitor. Subsequently, cells were washed three times with PBS containing $0.1 \%$ Tween-20 to avoid unspecific binding. The cellular fluorescence was measured with a FACS Aria III (BD, Heidelberg, Germany), using $488 \mathrm{~nm}$ as excitation wavelength, $530 \mathrm{~nm}$ for detection and the mean fluorescence value of at least 50,000 events for each measurement was determined.

Drugs affinity responsive target stability (DARTS): DARTS assay was performed to assess protease protection of Hsp90 protein from thermolysin digestion after incubation of Hsp90i, as described previously. ${ }^{19,28}$ Briefly, $1 \mu \mathrm{g}$ of recombinant Hsp90 $\alpha$ was incubated on ice for 15 min with $25,50 \&$ $100 \mu \mathrm{M}$ of $\mathbf{5 b}$, NB, AUY922 and DMSO. After incubation the samples were treated with thermolysin (1:50 of protein) for $5 \mathrm{~min}$. The reaction was halted by addition of $50 \mathrm{mM}$ EDTA and later examined by immunoblot analysis.

Luciferase refolding assay: Luciferase refolding assay was performed using recombinant firefly luciferase from Photinus pyralis (Sigma-Aldrich, St. Louis, MO, USA; 10x10 ${ }^{10}$ Units/mg), which was diluted (1:100) in denaturation buffer (25 mM Tricine, $\mathrm{pH}$ 7.8, $8 \mathrm{mM}$ MgSO4, $0.1 \mathrm{mM}$ EDTA, $1 \%$ Triton $\mathrm{X}-100,10 \%$ glycerol, and $10 \mathrm{mg} / \mathrm{mL}$ BSA) at $38^{\circ} \mathrm{C}$ for $8 \mathrm{~min} \cdot{ }^{42-44,78}$ Rabbit reticulocyte lysate (Promega, Madison, WI, USA) was diluted 1:1 by the addition of cold mix buffer (100 mM Tris, $\mathrm{pH}$ 7.7, $75 \mathrm{mM} \mathrm{Mg}(\mathrm{OAc}) 2,375 \mathrm{mM} \mathrm{KCl}$, and $15 \mathrm{mM} \mathrm{ATP})$, creatine phosphate $(10 \mathrm{mM})$ and creatine 
phosphokinase $(16 \mathrm{U} / \mathrm{mL})$ and was pre-incubated at $30^{\circ} \mathrm{C}$ with the respective inhibitors and controls for 1h. Afterwards, $1 \mu \mathrm{L}$ denatured luciferase or active luciferase (as a control) was added to $20 \mu \mathrm{L}$ rabbit reticulocyte mixture. As a control, denatured or active luciferase was incubated without reticulocyte lysate in buffer containing $20 \mathrm{mM}$ Tris, $\mathrm{pH} 7.5,150 \mathrm{mM} \mathrm{NaCl}, 1 \%$ haemoglobin and $4 \%$ BSA. At desired time points, $1.5 \mu \mathrm{L}$ samples were removed and added to $40 \mu \mathrm{L}$ assay buffer ( $25 \mathrm{mM}$ Tricine, $\mathrm{pH}$ 7.8, $8 \mathrm{mM} \mathrm{MgSO} 4,0.1 \mathrm{mM}$ EDTA, $33 \mu \mathrm{M}$ DTT, $0.5 \mathrm{mM}$ ATP, and $0.5 \mathrm{mM}$ luciferin) and luminescence was read using a Spark ${ }^{\circledR}$ microplate reader (Tecan). Percent luciferase refolding was determined using luminescence of DMSO at $120 \mathrm{~min}$ as $100 \%$ and comparing all samples to this value.

Thermal shift assay: CTD or NTD of r-Hsp90 $\alpha(5 \mu \mathrm{M})$ protein and the indicated concentrations of the inhibitors were mixed together in the assay buffer ( $1 \mathrm{X} \mathrm{PBS,} \mathrm{pH}=7.5)$ and were incubated for $2 \mathrm{~h}$. Then, 6X SYPRO Orange dye (Sigma-Aldrich) was added to the mixture $(20 \mu 1)$, which binds to the exposed hydrophobic residues of unfolded protein (during thermal exposure) and thereby showing an increase fluorescence. ${ }^{39,79}$ 96-well polymerase chain reaction (PCR) plates, and a PCR system (BioRad, CFX Connect ${ }^{\mathrm{TM}}$ Real-time system) were used to heat the samples from room temperature to $95{ }^{\circ} \mathrm{C}$ in increments of $0.5^{\circ} \mathrm{C}$ for 10 seconds, with excitation wavelength at $470 \mathrm{~nm}$ and emission wavelength at $570 \mathrm{~nm}$. For determination of protein melting temperature values $(\mathrm{Tm})$, melting curve for each data set was analysed by GraphPad Prism 8.0.2 and fitted with the sigmoidal Boltzmann fit. Melting temperatures without the inhibitors were used as control.

Isothermal shift assay was performed following similar procedure as with thermal shift assay. However, increasing indicated concentrations of the inhibitors $(98 \mathrm{nM}-200 \mu \mathrm{M})$ were used, which was incubated with either C-terminal or N-terminal of $\operatorname{Hsp} 90(5 \mu \mathrm{M})$ in the assay buffer (1X PBS, $\mathrm{pH}=7.5)$ for $2 \mathrm{~h}$. The samples were heated to $46^{\circ} \mathrm{C}$ after adding 6X SYPRO Orange dye (Sigma-Aldrich). Percent unfolding was calculated by normalizing to $100 \%$ unfolding and comparison to vehicle control (DMSO).

Cellular thermal shift assay (CETSA): CETSA assay was performed as described previously, ${ }^{39-41}$ with minor modifications. K562 cells were incubated with the indicated inhibitors (or DMSO) for 24h. Cells were harvested by centrifugation ( $400 \mathrm{x} \mathrm{g}$ for $5 \mathrm{~min}$ at RT) and washed three times with PBS. The pellets were dissolved in PBS and later equally divided into $200 \mu \mathrm{L}-\mathrm{PCR}$ tubes. Solutions were heated at the 
indicated temperature gradient for $3 \mathrm{~min}$ (T-Gradient Cycler, Biometra). Aliquots were then snap-freeze in liquid nitrogen and thawed at $25^{\circ} \mathrm{C}$ in a thermal cycler (GeneAMP PCR System2700, Applied Biosystems) three times, followed by centrifugation at $10000 \mathrm{x}$ g for $20 \mathrm{~min}$ at $4^{\circ} \mathrm{C}$. The supernatants were harvested and protein levels were measured by quantitative simple western immunoassay (JESS, BioTechne, MN, USA). Protein levels represented by the area under the curve of the electropherograms were normalized to the lowest temperature set as $0 \%$ degradation. $\Delta \mathrm{T}_{\mathrm{m}}$ for compounds were determined by plotting normalized data using sigmoid dose curve and nonlinear regression (GraphPad Prism 8.0.2).

Isothermal dose-dependent fingerprint (ITDRF) CETSA: Based on the previously determined IC50 values, K562 cells were treated with the indicated dilution range of inhibitor for $24 \mathrm{~h}$. Then, cells were harvested by centrifugation ( $400 \mathrm{x}$ g for $5 \mathrm{~min}$ at RT) and washed three times with PBS. The pellets were dissolved in $200 \mu \mathrm{L}$ PBS and transferred into PCR tubes (Eppendorf). Results of prior CETSA runs were utilized to determine the optimal melting temperature of $54.1^{\circ} \mathrm{C}$ for $\mathrm{Hsp} 90$. Samples were heated once at $54.1^{\circ} \mathrm{C}$ for $3 \mathrm{~min}$ (T-Gradient Cycler, Biometra) and then snap-frozen in liquid nitrogen and thawed at $25^{\circ} \mathrm{C}$ in a thermal cycler (GeneAMP PCR System2700, Applied Biosystems) three times, followed by centrifugation at $10000 \mathrm{xg}$ for $20 \mathrm{~min}$ at $4^{\circ} \mathrm{C}$. The supernatants were harvested and protein levels were measured by quantitative simple western immunoassay (JESS, BioTechne, MN, USA). Protein levels are calculated by the area under the curve of the electropherograms.

Simple western immunoassay: Fluorescent (5X) master mix, DTT and biotinylated ladder were prepared as per manufacturer's instruction (BioTechne). Lysates were diluted with $0.1 \mathrm{x}$ sample buffer and mixed 5:1 with fluorescent $5 \mathrm{x}$ master mix to obtain a target sample concentration of $0.40 \mu \mathrm{g} / \mu \mathrm{l}$ per well. Samples were then denatured for $5 \mathrm{~min}$ at $95^{\circ} \mathrm{C}$ in a PCR cycler (GeneAMP PCR System2700, Applied Biosystems). The assay plate was loaded as per manufacture's instruction and centrifuged for 5 min at $1000 \mathrm{xg}$ at RT. The immunoassay was performed using a 12-230 kDA separation module with 25 cartridges (SM-W004, BioTechne). Lysates were separated $25 \mathrm{~min}$ at 375 volt, blocked for 5 min with antibody diluent 2, 30 min incubated with primary antibody and 30 min incubated with secondary antibody, subsequently. Primary antibody multiplex mix consisted of 1:100 anti-Hsp90 (\#4877S, Cell Signaling Technology, Danvers, MA, USA) and 1:50 anti- $\beta$-actin (\#MAB8929, R\&D) diluted in 
Antibody Diluent 2. Signals were detected using an anti-rabbit detection module of JESS (DM-001, BioTechne), multiplexed with an anti-mouse secondary NIR antibody (\#043-821, BioTechne).

Time-resolved fluorescence resonance energy transfer (TR-FRET): The evaluation of the Hsp90 binding affinity to PPID (Cyclophilin D) was performed using the Hsp90 CTD TR-FRET assay kit (\#50289, BPS Bioscience, San Diego, CA, USA). Terbium (Tb) labeled donor and dye-labeled acceptor were diluted 100-fold in (1X) Hsp90 assay buffer 2. $10 \mu \mathrm{L}$ diluted tb-labeled donor and $10 \mu 1$ diluted dye-labeled acceptor were mixed with $4 \mu \mathrm{L}$ (indicated concentration) inhibitor, $10 \mu \mathrm{L}$ of $3 \mathrm{ng} / \mu 1$ PPIDGST-tag (BPS Bioscience) and $6 \mu \mathrm{L}$ of $2 \mathrm{ng} / \mu \mathrm{l}$ biotin labeled CTD of Hsp90 (BPS Biosciences). For the positive control, the inhibitor was substituted for DMSO and for the negative control PPID-GST-tag was substituted for $1 \mathrm{X}$ Hsp90 assay buffer. Samples were incubated for 2 hours at RT protected from light and measured with a microplate-reader (SPARK10M, Tecan). Fluorescence was measured using a time resolved reading mode with two subsequent measurement: First measurement was performed using $340 \mathrm{~nm} / 620 \mathrm{~nm}$ (excitation/emission) wavelength with a lag time of $60 \mu \mathrm{s}$ and integration time of 500 $\mu \mathrm{s}$. Second measurement was performed using $340 \mathrm{~nm} / 665 \mathrm{~nm}$ (excitation/emission) wavelength with a lag time of $60 \mu \mathrm{s}$ and integration time of $500 \mu \mathrm{s}$. Data analysis is performed using the TR-FRET ratio (665 $\mathrm{nm}$ emission / $620 \mathrm{~nm}$ emission). The TR-FRET ratios are normalized to \% activity by setting the negative control as zero percent activity and the positive control as one hundred percent activity $\left[\left(\mathrm{FRET}_{\text {sample }}-\mathrm{FRET}_{\text {neg }}\right) /\left(\mathrm{FRET}_{\text {pos }}-\mathrm{FRET}_{\text {neg }}\right) * 100 \%\right]$.

Fluorescence polarization (FP) measurements: The evaluation of the binding affinity of compounds towards the ATP pocket of Hsp90 NTD was determined by a competitive binding assay against FITClabeled geldanamycin (GM) using the Hsp90 NTD assay kit (\#50293, BPS Bioscience). ${ }^{42}$ The inhibitor sample wells were filled with $15 \mu 1$ XX Hsp90 assay buffer, $5 \mu \mathrm{L} 40 \mathrm{mM}$ DTT, $5 \mu \mathrm{L} 2 \mathrm{mg} / \mathrm{ml} \mathrm{BSA}, 40$ $\mu \mathrm{L} \mathrm{H}_{2} \mathrm{O}, 5 \mu \mathrm{L}$ FITC-labeled $\mathrm{GM}(100 \mathrm{nM})$ and $10 \mu \mathrm{L}$ inhibitor (at indicated concentration). The reaction was initiated by adding $20 \mu \mathrm{Hsp} 90(17 \mathrm{ng} / \mu \mathrm{l})$ and incubation at room temperature for 3 hours with slow shaking. Background wells (master mix only); negative controls (FITC-labeled GM, buffer and DMSO) and positive controls (FITC-labeled GM, buffer, DMSO, Hsp90) were also included within the assay plate. Fluorescence was measured at $470 \mathrm{~nm}$ excitation wavelength and $525 \mathrm{~nm}$ emission wavelength in a microtiter-plate reader (Infinite M1000pro by Tecan). Polarization was calculated using 
$\left(\mathrm{I}_{\mathrm{II}}-\mathrm{G}(\mathrm{I} \perp) /\left(\mathrm{I}_{\mathrm{II}}+\mathrm{G}(\mathrm{I} \perp)\right) * 1000\right.$ and a g-factor of 1,187. The percentage of Hsp90-bound FITC labelled GM was calculated using $\mathrm{P}_{\text {norm }}=\left(\mathrm{P}_{\text {Inhibitor }}-\mathrm{P}_{\text {neg }}\right) /\left(\mathrm{P}_{\text {pos }}-\mathrm{P}_{\text {neg }}\right) * 100$.

Molecular dynamics (MD) simulations: The structures of Hsp90 $\alpha$ and $\beta$ (PDB IDs $3 \mathrm{q} 6 \mathrm{~m}$ and $5 \mathrm{fwk}$ respectively) were prepared using Schrödinger Maestro ${ }^{80}$. For each of the isoforms, 40 individual MD simulations were performed. Initial random placement of $\mathbf{5 b}$ and solvation in TIP3P water $^{81}$ was done using PACKMOL ${ }^{82}$, neutralizing the system by the addition of sodium ions. ff14SB was used as force field for the protein and a modified GAFF version 1.5 for $\mathbf{5 b}{ }^{26,48}$ All simulations were carried out using the Amber 18 software package. ${ }^{46}$ To treat long-range electrostatics, the particle mesh Ewald method ${ }^{83}$ was used with a cutoff of $9.0 \AA$ for equilibration and $10.0 \AA$ for production. The SHAKE algorithm ${ }^{84}$ and hydrogen mass repartitioning ${ }^{85}$ were used to allow for simulation steps of 2 fs in the equilibration and $4 \mathrm{fs}$ in the production.

Initially, the systems were energy-minimized using the steepest descent (500 steps) and conjugate gradient (2000 steps) methods and placing positional restraints with a force constant of $5 \mathrm{kcal} \mathrm{mol}^{-1} \AA^{-2}$ on all protein atoms; the restraints were reduced in a second energy minimization to a force constant of $1 \mathrm{kcal} \mathrm{mol}{ }^{-1} \AA^{-2}$ (for 2000 steps of steepest descent followed by 8000 steps of conjugate gradient), and removed in a third one (for 1000 steps of steepest descent followed by 4000 steps of conjugate gradient). Placing positional restraints with a force constant of $1 \mathrm{kcal} \mathrm{mol}^{-1} \AA^{-2}$ on the backbone atoms, first, the system was heated to $100 \mathrm{~K}$ in $50 \mathrm{ps}$ of NVT MD and further heated to $300 \mathrm{~K}$ in 5 ps of NPT MD. Further 65 ps of NPT MD were performed for density equilibration, still applying the backbone restraints. Afterward, over the course of further 300 ps of NPT MD, the backbone restraints were gradually reduced to a force constant of $0.2 \mathrm{kcal} \mathrm{mol}^{-1} \AA^{-2}$.

After the thermalization, $500 \mathrm{~ns}$ of NPT MD simulations were carried out with positional restraints with a force constant of $0.1 \mathrm{kcal} \mathrm{mol}^{-1} \AA^{-2}$ on the backbone atoms. The Langevin thermostat with a collision frequency of $1 \mathrm{ps}^{-1}$ and the Monte Carlo barostat with a pressure relaxation time of $1 \mathrm{ps}$ were used. The reference coordinates for these restraints were readjusted every $100 \mathrm{~ns}$ to allow for moderate protein flexibility. For Hsp90a, these simulations were later extended to $1 \mu \mathrm{s}$. The trajectories were postprocessed and analyzed with CPPTRAJ ${ }^{86}$, and results were visualized with PyMol $^{76}$. 
MM-GB/SA computations: Effective binding energies were computed over one MD trajectory that resulted in binding of $\mathbf{5 b}$ in the CTD dimerization interface and led to a binding mode in which $\mathbf{5 b}$ mimics H5'. In the computations, the single trajectory approach was used, where complex, protein, and ligand configurations were extracted from the complex trajectory. ${ }^{87}$ After removing water molecules and counterions, gas-phase energies (van der Waals and electrostatic contributions) were evaluated on every frame sampled at an interval of 200 ps using MMPBSA.py ${ }^{88}$; the polar contribution to the solvation free energy was calculated using the "OBC II" generalized Born model ${ }^{89}$, together with mbondi 2 radii and a dielectric constant of 1 for the solute and 80 for the solvent. The ionic strength was set to $150 \mathrm{mM}$ of a 1:1 salt. The non-polar contribution to the solvation free energy was calculated as a function of the solvent-accessible surface area using $0.0050 \mathrm{kcal} \mathrm{mol}^{-1} \AA^{-2}$ as surface tension. No absolute binding free energies can be derived from this approach since configurational entropy contributions are not considered here to reduce the uncertainty in the computations. ${ }^{49,50}$. However, the sum of gas-phase energies and solvation free energies, the effective energy, is suitable to estimate relative differences in binding free energies for differential binding poses. ${ }^{28}$

WB \& Blue-native gels: Cells were treated with the indicated concentration of the compound or vehicle (DMSO) for 48 hours. Immunoblot analysis was performed by following standard protocol using antibodies: anti-Hsp90 (\#4877), anti-Hsp70 (\#4872), anti-Hsp40 (\#4871), anti-Hsp27 (\#2402), anti-

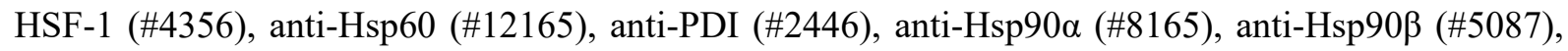
anti-c-Abl (\#2862), anti-phospho-c-Abl (\#2865), anti-Stat5 (\#9363), anti-phospho-Stat5 (\#9351), antiCrkL (\#3182), anti-phospho-Crkl (\#3181), anti-Akt (\#2920), anti-phospho-Akt (\#4060), anti-S6 ribosomal protein (\#2217), anti-phospho-S6 ribosomal protein (\#4858), anti-c-Myc (\#13987) from Cell Signaling Technology and anti- $\beta$-actin (Sigma-Aldrich). Blue-native (BN) gels were performed following manufacturer's instructions (Invitrogen) and performed previously. ${ }^{28}$ Briefly, lysates were generated from K562 cell line after $48 \mathrm{~h}$ treatment with inhibitors (at indicated concentration) using NativePAGE Sample Prep kit (Invitrogen) by 2-3 freezing thawing cycles followed by centrifugation at $20,000 \mathrm{x}$ g for $25-30 \mathrm{~min}$ at $4^{\circ} \mathrm{C}$.

Dimerization assay: Hsp90 CTD dimerization was evaluated using an amine-reactive chemical crosslinker bis(sulfosuccinimidyl) suberate $\left(\mathrm{BS}^{3}\right)$ (Pierce). ${ }^{42,45}$ Hsp90 $\alpha$ CTD protein $(2 \mu \mathrm{M})$ was diluted in 
$\mathrm{Na}_{2} \mathrm{HPO}_{4}(25 \mathrm{mM} ; \mathrm{pH} 7.4)$ and treated with different concentrations of the inhibitor to make a final volume of $25 \mu \mathrm{L}$. The reaction mixture was incubated at RT for $1 \mathrm{~h}$. The amine-reactive crosslinker $\mathrm{BS}^{3}$ was added to a final concentration of $63 \mu \mathrm{M}$ and the samples were incubated for $1 \mathrm{~h}$ at RT. Crosslinking was quenched by the addition of SDS sample buffer and subsequent heating for $5 \mathrm{~min}$ at $95{ }^{\circ} \mathrm{C}$. Samples were run in $12 \%$ SDS-PAGE gels followed by western blotting. Blots were probed with anti-Hsp90 (AC88, Abcam) antibody.

SEC-SAXS: We collected the SEC-SAXS data on beamline BM29 at the ESRF Grenoble ${ }^{53,54}$. The BM29 beamline was equipped with a PILATUS 2M detector (Dectris) at a fixed distance of $2.827 \mathrm{~m}$. The measurement of $\mathrm{Hsp} 90 \mathrm{CTD}(18 \mathrm{mg} / \mathrm{ml})$ was performed at $20^{\circ} \mathrm{C}$ on a Superdex 200 increase $3.2 / 300$ column (Buffer $50 \mathrm{mM}$ TRIS pH 7.5, $100 \mathrm{mM} \mathrm{NaCl}$ ) with a flowrate of $0.075 \mathrm{ml} / \mathrm{min}$, collecting one frame each two seconds. Data were scaled to absolute intensity against water. Further, we have collected SAXS data on our Xeuss 2.0 Q-Xoom sytem from Xenocs, equipped with a PILATUS 3 R 300K detector (Dectris) and a GENIX 3D CU Ultra Low Divergence x-ray beam delivery system. The chosen sample to detector distance for the experiment was $0.55 \mathrm{~m}$, results in an achievable q-range of $0.10-6 \mathrm{~nm}^{-1}$. All measurements were performed at $20^{\circ} \mathrm{C}$ with protein concentrations of 9.7 and $10.8 \mathrm{mg} / \mathrm{mL}$. Compound 5 b was added and incubated for $30 \mathrm{~min}$ at $20^{\circ} \mathrm{C}$. Samples were injected in the Low Noise Flow Cell (Xenocs) via autosampler. For each sample, 18 frames with an exposer time of ten minutes were collected. Data were scaled to absolute intensity against water. All used programs for data processing were part of the ATSAS Software package (Version 3.0.3) ${ }^{90}$. Primary data reduction was performed with the programs CHROMIXS and PRIMUS ${ }^{55,56}$. With the Guinier approximation ${ }^{58}$, we determine the forward scattering $I(0)$ and the radius of gyration $\left(R_{g}\right)$. The program GNOM ${ }^{91}$ was used to estimate the maximum particle dimension $\left(D_{\max }\right)$ with the pair-distribution function $p(r)$. Low resolution ab initio models were calculated with DAMMIF ${ }^{57}$. Superimposing of the predicted model was done with the program SUPCOMB ${ }^{92}$.

Physicochemical properties of $\mathbf{5 b}$ (See Supplementary Note 2 for more details): Aqueous solubility of $\mathbf{5 b}$

The aqueous thermodynamic solubility of $\mathbf{5 b}$ was determined in phosphate-buffered-saline (PBS, $\mathrm{pH}$ 7.4) after 4 and $24 \mathrm{~h}$ incubation time at $25^{\circ} \mathrm{C}$. Ondansetron was used as reference compound with high 
solubility of $95 \mu \mathrm{M}$. The thermodynamic solubility of $\mathbf{5 b}$ was ranging from $4 \mu \mathrm{M}$ after $4 \mathrm{~h}$ to $8 \mu \mathrm{M}$ after $24 \mathrm{~h}(\mathrm{n}=2)$. For detailed information see Bienta, Enamie Biological Services study reports.

\section{Chemical stability of $\mathbf{5 b}$}

Drug decomposition was determined by high-performance liquid chromatography (HPLC, Method 1). Instrument: Knauer HPLC system in combination with a Knauer UV Detector Azura UVD 2.1L. Column: KNAUER Eurospher II 100-5 C18, 150x4 mm Mobile phase 1: linear gradient (90-0\%) of water with $0.1 \%$ trifluoroacetic acid. Mobile phase 2: linear gradient (10-100\%) of acetonitrile with $0.1 \%$ of trifluoroacetic acid. Run time: $20 \mathrm{~min}$, followed by an isocratic elution with $100 \%$ acetonitrile for $10 \mathrm{~min}$. Flow rate: $1 \mathrm{~mL} / \mathrm{min}$. Detection: $254 \mathrm{~nm}$.

5b was dissolved in a mixture of Tween20/ethanol/phosphate buffer $\mathrm{pH} 7,4(7 / 3 / 90)$ and the stability monitored over a period of $24 \mathrm{~h}$ at $37^{\circ} \mathrm{C}$. After $24 \mathrm{~h}$, almost no decomposition was detected $(0.7 \% \mathrm{drug}$ decomposition, $n=2$ ).

The stability of $\mathbf{5 b}$ at acidic $\mathrm{pH}$ was determined by dissolving $\mathbf{5 b}$ in a mixture of Tween20/ethanol/phosphate buffer $\mathrm{pH} 2$ (7/3/90) and the stability monitored over a period of $24 \mathrm{~h}$ at $37^{\circ} \mathrm{C}$. After $24 \mathrm{~h}$, only slight decomposition was detected $(1.3 \%$ drug decomposition, $n=2)$.

\section{In vitro metabolic stability of $\mathbf{5 b}$ in human liver microsomes}

The metabolic stability screening of $\mathbf{5 b}$ in human liver microsomes revealed $\mathbf{9 1} \%$ stability after a 40 min incubation at $37^{\circ} \mathrm{C}$. Propanolol, a reference drug with medium to high metabolic stability showed $74 \%$ of the parent compound remaining, and therefore demonstrated slightly decreased stability compared with $\mathbf{5 b}$. Calculated results for the intrinsic clearance suggest that $\mathbf{5 b}(6 \mu \mathrm{L} / \mathrm{min} / \mathrm{mg})$ is a low clearance compound with an estimated long half-life $(n=2)$. For detailed information see Bienta, Enamie Biological Services study reports.

Cell culture: K562, KCL22, SUPB15 (BCR-ABL1+ CML/BCP-ALL), HL-60 (AML), DND41, HPBALL, TALL1 and MOLT4 (T-ALL) leukemic cell lines (DSMZ, Braunschweig, Germany) were cultured in RPMI1640 GlutaMAX (ThermoFisher Scinetific) supplemented with 10-20\% FCS (SigmaAldrich, St. Louis, MO, USA) and penicillin/streptomycin (Invitrogen, Carlsbad, CA, USA), and maintained at $37^{\circ} \mathrm{C}$ with $5 \% \mathrm{CO}_{2}$. Normal $\mathrm{BA} / \mathrm{F} 3$ and $\mathrm{BA} / \mathrm{F} 3$ cells expressing BCR-ABL1 mutants (T315I, M351T \& E255K) (murine pro B cell line) were cultured in RPMI1640 GlutaMAX (10\% FCS) supplemented with or without IL-3 (10ng/ml) respectively. BA/F3 cells expressing BCR-ABL1 mutants were resistant against imatinib (IM) until $\sim 10 \mu \mathrm{M} .^{28}$ IM resistant BCR-ABL1+ K562 (K562-IMr), KCL22 (KCL22-IMr) and SUPB15 (SUPB15-IMr) were generated by gradual increase $(1-2.5 \mu \mathrm{M})$ in 
the concentration of IM (Sigma-Aldrich, St. Louis, MO, USA) over a period of 3 months. ${ }^{28,93}$ Bortezomib (BTZ) resistant clones (80 nM) of HL60 (HL60-BTZr) was established following similar protocol as described to pick IM resistant clones. Primary patient derived $\mathrm{CML}^{\mathrm{CD} 34+}$ blast cells were cultured in mononuclear cell medium (PromoCell, Heidelberg, Germany).

Hsp90 CTD/NTD targeting reference inhibitors: Coumermycin A1 (CA1) and novobiocin (NB) were purchased from Sigma-Aldrich, and Hsp90 NTD targeting reference control inhibitors: geldanamycin (GM), tanespimycin (TM), PUH-71 were purchased from Selleckchem.

Viability assay: Cells were seeded in white 96-well plate (Corning, NY, USA) with increasing concentration $(50 \mathrm{nM}-25 \mu \mathrm{M})$ of inhibitors and respective controls for $48 \mathrm{~h}$. Cell viability was monitored using Celltitre Glo luminescent assay (based on the ATP quantification), following manufacturer's guidelines (Promega) ${ }^{28}{ }^{2} C_{50}$ for compounds were determined by plotting raw data (normalized to controls) using sigmoid dose curve and nonlinear regression (GraphPad Prism).

Proliferation assay: Cell proliferation was examined after treatment with the compounds at their indicated concentration with trypan exclusion method using automated cell counter (Vi-CELL TM XR Beckman Coulter, Fullerton, CA), after every $24 \mathrm{~h}$ interval.

Annexin V staining: For evaluating apoptosis, $48 \mathrm{~h}$ inhibitor treated cells were stained with Annexin $\mathrm{V}$ and propidium iodide (PI), following supplier's guidelines (Invitrogen, Carlsbad, CA) and later the stained cells were subjected to FACS (Cytoflex, Beckman Coulter).

Caspase 3/7 Glo assay: Cells were incubated with the respective inhibitors or control for $48 \mathrm{~h}$ and later enzymatic activity of caspase 3/7 was examined (SPARK10M, Tecan) by using caspase 3/7 dependent Glo assay (absorbance at $405 \mathrm{~nm}$ ), following manufacturer's instructions (Promega). ${ }^{28}$

Zebrafish: Zebrafish wild-type TE strain were maintained according to standard protocols and handled in accordance with European Union animal protection directive 2010/63/EU and the local government (Tierschutzgesetz §11, Abs. 1, Nr. 1, husbandry permit 35/9185.46/Uni TÜ). All experiments described in this study were conducted on embryos younger than 5 days post-fertilization (dpf) - prior to the legal onset of animal life. 
Xenotransplantation in zebrafish embryos: Xenotransplantation experiment was performed as described previously. ${ }^{65}$ Briefly, MOLT-4 cells were labeled with Vybrant ${ }^{\mathrm{TM}}$ CFDA SE Cell Tracer Kit (Invitrogen) following the manufacturer's instructions, and then were suspended in PBS at a density of $1 \times 10^{8} \mathrm{cells} / \mathrm{ml}$. Approximately $1 \mathrm{nl}$ cell suspension (around $200 \mathrm{cells}$ ) was injected into the perivitelline space of embryos at $32 \mathrm{hpf}$. Injected embryos were first incubated at $28^{\circ} \mathrm{C}$ for one hour. Only embryos with good engraftment were selected for treatment with DMSO (control group), AUY922 (500 nM) or $5 \mathrm{~b}(500 \mathrm{nM})$ for 48 hours at $35^{\circ} \mathrm{C}$. This temperature enables the maintenance of embryos with grafted cells without compromising zebrafish development. Drug-treated embryos were dissociated by passing through a 40- $\mu \mathrm{m}$ cell strainer (Greiner Bio-One) and then analyzed using a BD LSR II flow cytometer. Fold change of engrafted MOLT4 cells was calculated to mean of DMSO-treated embryos. GraphPad Prism software (version 7) was used for graphing and statistical analysis.

Differentiation assay: FACS measurements were performed after incubating the respective inhibitors at indicated concentration for $48 \mathrm{~h}$ by using fluorochrome coupled monoclonal antibodies (mAbs) along with matched isotype controls: anti-CD11b (Bear1; Beckman Coulter), anti-CD14 (RMO52; Beckman Coulter), anti-CD133 (AC133; MiltenyiBiotec, Gladbach, Germany). ${ }^{38}$

Colony forming unit (CFU) assay: CFU assay was performed by plating inhibitor treated cells (48 h) in methylcellulose medium (Methocult H4100 - StemCell Technologies, Vancouver, BC, Canada) supplemented with 50 ng/ml SCF, 10 ng/ml IL-3, 10 ng/ml GM-CSF (Peprotech, Hamburg, Germany) and $3 \mathrm{U} / \mathrm{ml}$ erythropoietin (ebiosciences, San Diego, USA). ${ }^{38}$ Colonies were counted after 14 days $(n=3)$.

Significance analyses of normally distributed data with variance similar between groups used paired, two-tailed Student's t-test: *, $\mathrm{p}<0.05 ; * *, \mathrm{p}<0.005 ;{ }^{* * *}, \mathrm{p}<0.001$. 


\section{Author Contributions:}

Conceptualisation: H.G., T.K., S.B. and L.S.; development and application of methodology: D.B., N.D., M.V., B.F., H.P., B.L., V.W., F.L., M.G., J.L., B.B., D.D., N.A., Ba.B., J.S., J.S-D., F.K.H, J.J., J.H., A.B., S.B., H.G., T.K.; analysis of data: D.B., B.F., J.L., H.G., S.B., N.D., M.V., V.W., T.K. and L.S.; S.S and J.R. performed and analyzed the SAXS experiment; manuscript writing: S.B., H.G., T.K., D.B., V.W. The study was supervised by H.G., T.K., and S.B.

\section{Funding Sources:}

This study was funded in part by the Deutsche Forschungsgemeinschaft (DFG, German Research Foundation) - 270650915 (Research Training Group GRK 2158: TP4a to H.G., TP4b to S.S., TP2c to T.K., and TP2d to S.B.). S.B., H.G., and T.K. are supported by Forschungskommission and DSONetzwerkverbundes, HHU Düsseldorf. S.B. additionally acknowledges the financial support by KinderKrebsForschung e.V. J.H. and A.B. have been supported by the TransOnc priority program of the German Cancer Aid within grant \#70112951 (ENABLE). JH has been supported by ERC Stg 85222 "PreventALL" and ERA PerMED 2018 "GEPARD". T.K. and H.G. were supported by funds from the Strategischer Forschungsfonds of HHU. The Center for Structural studies is funded by the DFG (Grant number 417919780 and INST 208/761-1 FUGG to S.S.).

\section{Notes:}

The authors declare no competing financial interest.

\section{Acknowledgments:}

We are grateful for computational support by the "Zentrum für Informations und Medientechnologie" at the Heinrich-Heine-Universität Düsseldorf and the computing time provided by the John von Neumann Institute for Computing (NIC) to H.G. on the supercomputer JUWELS at Jülich Supercomputing Centre (JSC) (user IDs: HKF7, VSK33). A.B. acknowledges the financial support of Katharina-Hardt Foundation, and Löwenstern e.V. for providing a Simple Western Instrument (JESS). 
We thank Anton Popov for the great support at BM29, as well as the whole ESRF Outstation Grenoble Team.

\section{Abbreviations:}

TLC, thin-layer chromatography; MOA mode of action; MD molecular dynamics; MM-GB/SA molecular mechanics generalized Born surface area; NTD N-terminal domain; MD Middle domain; CTD C-terminal domain; PPIs, protein-protein interactions; HSR, Heat shock resposne. 


\section{References}

1 Sanchez, J., Carter, T. R., Cohen, M. S. \& Blagg, B. S. Old and New Approaches to Target the Hsp90 Chaperone. Current cancer drug targets, doi:10.2174/1568009619666191202101330 (2019).

2 Schopf, F. H., Biebl, M. M. \& Buchner, J. The HSP90 chaperone machinery. Nature reviews. Molecular cell biology 18, 345-360, doi:10.1038/nrm.2017.20 (2017).

3 Butler, L. M., Ferraldeschi, R., Armstrong, H. K., Centenera, M. M. \& Workman, P. Maximizing the therapeutic potential of HSP90 inhibitors. Molecular cancer research : MCR 13, 1445-1451, doi:10.1158/1541-7786.MCR-15-0234 (2015).

4 Wang, Y., Koay, Y. C. \& McAlpine, S. R. How Selective are Hsp90 Inhibitors for Cancer Cells over Normal Cells? ChemMedChem 12, 353-357, doi:10.1002/cmdc.201600595 (2017).

$5 \quad$ Wu, J. et al. Heat Shock Proteins and Cancer. Trends Pharmacol Sci 38, 226-256, doi:10.1016/j.tips.2016.11.009 (2017).

6 Wang, Y. \& McAlpine, S. R. Heat-shock protein 90 inhibitors: will they ever succeed as chemotherapeutics? Future Med Chem 7, 87-90, doi:10.4155/fmc.14.154 (2015).

7 Hsu, H. S. et al. Chemoresistance of lung cancer stemlike cells depends on activation of Hsp27. Cancer 117, 1516-1528, doi:10.1002/cncr.25599 (2011).

8 Gandhi, N. et al. Novel Hsp90 inhibitor NVP-AUY922 radiosensitizes prostate cancer cells. Cancer biology \& therapy 14, 347-356, doi:10.4161/cbt.23626 (2013).

9 Modi, S. et al. A multicenter trial evaluating retaspimycin HCL (IPI-504) plus trastuzumab in patients with advanced or metastatic HER2-positive breast cancer. Breast cancer research and treatment 139, 107-113, doi:10.1007/s10549-013-2510-5 (2013).

10 Wang, M. et al. Development of Heat Shock Protein (Hsp90) Inhibitors To Combat Resistance to Tyrosine Kinase Inhibitors through Hsp90-Kinase Interactions. J Med Chem, doi:10.1021/acs.jmedchem.5b01106 (2016).

11 Bagatell, R. et al. Induction of a heat shock factor 1-dependent stress response alters the cytotoxic activity of hsp90-binding agents. Clin Cancer Res 6, 3312-3318 (2000).

12 McCollum, A. K., Teneyck, C. J., Sauer, B. M., Toft, D. O. \& Erlichman, C. Upregulation of heat shock protein 27 induces resistance to 17 -allylaminodemethoxygeldanamycin through a glutathione-mediated mechanism. Cancer research 66, 10967-10975, doi:10.1158/0008-5472.CAN-06-1629 (2006).

13 Guo, F. et al. Abrogation of heat shock protein 70 induction as a strategy to increase antileukemia activity of heat shock protein 90 inhibitor 17-allylamino-demethoxy geldanamycin. Cancer research 65, 10536-10544, doi:10.1158/0008-5472.CAN-051799 (2005).

14 Maloney, A. et al. Gene and protein expression profiling of human ovarian cancer cells treated with the heat shock protein 90 inhibitor 17-allylamino-17demethoxygeldanamycin. Cancer research 67, 3239-3253, doi:10.1158/00085472.CAN-06-2968 (2007).

15 Hall, J. A., Forsberg, L. K. \& Blagg, B. S. Alternative approaches to Hsp90 modulation for the treatment of cancer. Future Med Chem 6, 1587-1605, doi:10.4155/fmc.14.89 (2014).

16 Wang, Y. \& McAlpine, S. R. N-terminal and C-terminal modulation of Hsp90 produce dissimilar phenotypes. Chemical communications 51, 1410-1413, doi:10.1039/c4cc07284g (2015).

17 Li, L., Wang, L., You, Q. D. \& Xu, X. L. Heat Shock Protein 90 Inhibitors: An Update on Achievements, Challenges, and Future Directions. J Med Chem 63, 17981822, doi:10.1021/acs.jmedchem.9b00940 (2020). 
18 Khandelwal, A. et al. Structure-guided design of an Hsp90beta N-terminal isoformselective inhibitor. Nat Commun 9, 425, doi:10.1038/s41467-017-02013-1 (2018).

19 Eskew, J. D. et al. Development and characterization of a novel C-terminal inhibitor of Hsp90 in androgen dependent and independent prostate cancer cells. BMC Cancer 11, 468, doi:10.1186/1471-2407-11-468 (2011).

20 Koay, Y. C. et al. Chemically accessible hsp90 inhibitor that does not induce a heat shock response. ACS medicinal chemistry letters 5, 771-776, doi:10.1021/ml500114p (2014).

21 Sellers, R. P. et al. Design and synthesis of Hsp90 inhibitors: exploring the SAR of Sansalvamide A derivatives. Bioorganic \& medicinal chemistry 18, 6822-6856, doi:10.1016/j.bmc.2010.07.042 (2010).

22 Westerheide, S. D. et al. Celastrols as inducers of the heat shock response and cytoprotection. J Biol Chem 279, 56053-56060, doi:10.1074/jbc.M409267200 (2004).

23 Bickel, D. \& Gohlke, H. C-terminal modulators of heat shock protein of $90 \mathrm{kDa}$ (HSP90): State of development and modes of action. Bioorganic \& medicinal chemistry 27, 115080, doi:10.1016/j.bmc.2019.115080 (2019).

24 Metz, A. et al. Hot spots and transient pockets: predicting the determinants of smallmolecule binding to a protein-protein interface. J Chem Inf Model 52, 120-133, doi:10.1021/ci200322s (2012).

25 Metz, A., Schanda, J., Grez, M., Wichmann, C. \& Gohlke, H. From determinants of RUNX1/ETO tetramerization to small-molecule protein-protein interaction inhibitors targeting acute myeloid leukemia. J Chem Inf Model 53, 2197-2202, doi:10.1021/ci400332e (2013).

26 Ciglia, E. et al. Resolving hot spots in the C-terminal dimerization domain that determine the stability of the molecular chaperone Hsp90. PloS one 9, e96031, doi:10.1371/journal.pone.0096031 (2014).

27 Bopp, B. et al. Design and biological testing of peptidic dimerization inhibitors of human Hsp90 that target the C-terminal domain. Biochimica et biophysica acta $\mathbf{1 8 6 0}$, 1043-1055, doi:10.1016/j.bbagen.2016.01.005 (2016).

28 Bhatia, S. et al. Targeting HSP90 dimerization via the $\mathrm{C}$ terminus is effective in imatinib-resistant CML and lacks the heat shock response. Blood 132, 307-320, doi:10.1182/blood-2017-10-810986 (2018).

29 Krieger, V. et al. alpha-Aminoxy Peptoids: A Unique Peptoid Backbone with a Preference for cis-Amide Bonds. Chemistry 23, 3699-3707, doi:10.1002/chem.201605100 (2017).

30 Spanier, L. et al. Design, synthesis, and conformational analysis of trispyrimidonamides as alpha-helix mimetics. The Journal of organic chemistry 79, 1582-1593, doi:10.1021/jo402353z (2014).

31 Verba, K. A. et al. Atomic structure of Hsp90-Cdc37-Cdk4 reveals that Hsp90 traps and stabilizes an unfolded kinase. Science 352, 1542-1547, doi:10.1126/science.aaf5023 (2016).

32 Marcu, M. G., Chadli, A., Bouhouche, I., Catelli, M. \& Neckers, L. M. The heat shock protein 90 antagonist novobiocin interacts with a previously unrecognized ATPbinding domain in the carboxyl terminus of the chaperone. J Biol Chem 275, 3718137186, doi:DOI 10.1074/jbc.M003701200 (2000).

33 Eccles, S. A. et al. NVP-AUY922: A novel heat shock protein 90 inhibitor active against xenograft tumor growth, angiogenesis, and metastasis. Cancer research $\mathbf{6 8}$, 2850-2860, doi:10.1158/0008-5472.Can-07-5256 (2008).

34 Yin, Z. Y., Henry, E. C. \& Gasiewicz, T. A. (-)-Epigallocatechin-3-gallate Is a Novel Hsp90 Inhibitor. Biochemistry-Us 48, 336-345, doi:10.1021/bi801637q (2009). 
35 Lundgren, K. et al. BIIB021, an orally available, fully synthetic small-molecule inhibitor of the heat shock protein Hsp90. Mol Cancer Ther 8, 921-929, doi:10.1158/1535-7163.MCT-08-0758 (2009).

36 Pacey, S. et al. A Phase II trial of 17-allylamino, 17-demethoxygeldanamycin (17AAG, tanespimycin) in patients with metastatic melanoma. Invest New Drugs 30, 341 349, doi:10.1007/s10637-010-9493-4 (2012).

37 Jose, J. \& von Schwichow, S. Autodisplay of active sorbitol dehydrogenase (SDH) yields a whole cell biocatalyst for the synthesis of rare sugars. Chembiochem 5, 491499, doi:10.1002/cbic.200300774 (2004).

38 Bhatia, S. et al. Control of AC133/CD133 and impact on human hematopoietic progenitor cells through nucleolin. Leukemia 29, 2208-2220, doi:10.1038/leu.2015.146 (2015).

39 Wang, L. et al. Discovery and Optimization of Small Molecules Targeting the ProteinProtein Interaction of Heat Shock Protein 90 (Hsp90) and Cell Division Cycle 37 as Orally Active Inhibitors for the Treatment of Colorectal Cancer. Journal of medicinal chemistry 63, 1281-1297, doi:10.1021/acs.jmedchem.9b01659 (2020).

40 Liang, C. et al. Design and synthesis of N-(5-chloro-2,4-dihydroxybenzoyl)-(R)1,2,3,4-tetrahydroisoquinoline-3-carboxamid es as novel Hsp90 inhibitors. European journal of medicinal chemistry 121, 272-282, doi:10.1016/j.ejmech.2016.05.033 (2016).

41 Jafari, R. et al. The cellular thermal shift assay for evaluating drug target interactions in cells. Nat Protoc 9, 2100-2122, doi:10.1038/nprot.2014.138 (2014).

42 Goode, K. M. et al. Targeting the Hsp90 C-terminal domain to induce allosteric inhibition and selective client downregulation. Biochimica et biophysica acta. General subjects 1861, 1992-2006, doi:10.1016/j.bbagen.2017.05.006 (2017).

43 McConnell, J. R., Alexander, L. A. \& McAlpine, S. R. A heat shock protein 90 inhibitor that modulates the immunophilins and regulates hormone receptors without inducing the heat shock response. Bioorg Med Chem Lett 24, 661-666, doi:10.1016/j.bmcl.2013.11.059 (2014).

44 Galam, L. et al. High-throughput assay for the identification of Hsp90 inhibitors based on Hsp90-dependent refolding of firefly luciferase. Bioorganic \& medicinal chemistry 15, 1939-1946, doi:10.1016/j.bmc.2007.01.004 (2007).

45 Allan, R. K., Mok, D., Ward, B. K. \& Ratajczak, T. Modulation of chaperone function and cochaperone interaction by novobiocin in the C-terminal domain of Hsp90: evidence that coumarin antibiotics disrupt Hsp90 dimerization. J Biol Chem 281, 7161-7171, doi:10.1074/jbc.M512406200 (2006).

46 AMBER 2018 (University of California, San Francisco, 2018).

47 James, A. M. et al. ff14SB: Improving the accuracy of protein side chain and backbone parameters from ff99SB. Journal of Chemical Theory and Computation 11, 3696-3713 (2015).

48 Wang, J., Wolf, R. M., Caldwell, J. W., Kollman, P. A. \& Case, D. A. Development and testing of a general amber force field. J Comput Chem 25, 1157-1174, doi:10.1002/jcc.20035 (2004).

49 Sun, H. Y. et al. Assessing the performance of MM/PBSA and MM/GBSA methods. 7. Entropy effects on the performance of end-point binding free energy calculation approaches. Phys Chem Chem Phys 20, 14450-14460, doi:10.1039/c7cp07623a (2018).

50 Gohlke, H. \& Case, D. A. Converging free energy estimates: MM-PB(GB)SA studies on the protein-protein complex Ras-Raf. Journal of Computational Chemistry 25, 238250, doi:10.1002/jcc.10379 (2004). 
51 Pearl, L. H. \& Prodromou, C. Structure and mechanism of the Hsp90 molecular chaperone machinery. Annual review of biochemistry 75, 271-294, doi:10.1146/annurev.biochem.75.103004.142738 (2006).

52 Wayne, N. \& Bolon, D. N. Dimerization of Hsp90 is required for in vivo function. Design and analysis of monomers and dimers. J Biol Chem 282, 35386-35395, doi:10.1074/jbc.M703844200 (2007).

53 Pernot, P. et al. New beamline dedicated to solution scattering from biological macromolecules at the ESRF. Journal of Physics: Conference Series 247, 012009 (2010).

54 Pernot, P. et al. Upgraded ESRF BM29 beamline for SAXS on macromolecules in solution. J Synchrotron Radiat 20, 660-664, doi:10.1107/S0909049513010431 (2013).

55 Panjkovich, A. \& Svergun, D. I. CHROMIXS: automatic and interactive analysis of chromatography-coupled small angle X-ray scattering data. Bioinformatics, doi:10.1093/bioinformatics/btx846 (2017).

56 Konarev, P. V., Volkov, V. V., Sokolova, A. V., Koch, M. H. J. \& Svergun, D. I. PRIMUS: a Windows PC-based system for small-angle scattering data analysis. $J$ Appl Crystallogr 36, 1277-1282, doi:10.1107/S0021889803012779 (2003).

57 Franke, D. \& Svergun, D. I. DAMMIF, a program for rapid ab-initio shape determination in small-angle scattering. J Appl Crystallogr 42, 342-346, doi:10.1107/S0021889809000338 (2009).

58 Guinier, A. Diffraction of x-rays of very small angles-application to the study of ultramicroscopic phenomenon. Annales de Physique 12, 161-237 (1939).

59 Svergun, D., Barberato, C. \& Koch, M. H. J. CRYSOL - A program to evaluate x-ray solution scattering of biological macromolecules from atomic coordinates. J Appl Crystallogr 28, 768-773, doi:Doi 10.1107/S0021889895007047 (1995).

60 Mahalingam, D. et al. Targeting HSP90 for cancer therapy. British journal of cancer 100, 1523-1529, doi:10.1038/sj.bjc.6605066 (2009).

61 Khajapeer, K. V. \& Baskaran, R. Hsp90 Inhibitors for the Treatment of Chronic Myeloid Leukemia. Leukemia research and treatment 2015, 757694, doi:10.1155/2015/757694 (2015).

62 Lazenby, M., Hills, R., Burnett, A. K. \& Zabkiewicz, J. The HSP90 inhibitor ganetespib: A potential effective agent for Acute Myeloid Leukemia in combination with cytarabine. Leukemia research 39, 617-624, doi:10.1016/j.leukres.2015.03.016 (2015).

63 Miyata, Y., Nakamoto, H. \& Neckers, L. The therapeutic target Hsp90 and cancer hallmarks. Current pharmaceutical design 19, 347-365 (2013).

64 Lu, X., Xiao, L., Wang, L. \& Ruden, D. M. Hsp90 inhibitors and drug resistance in cancer: the potential benefits of combination therapies of Hsp90 inhibitors and other anti-cancer drugs. Biochemical pharmacology 83, 995-1004, doi:10.1016/j.bcp.2011.11.011 (2012).

65 Morishima, T. et al. LMO2 activation by deacetylation is indispensable for hematopoiesis and T-ALL leukemogenesis. Blood 134, 1159-1175, doi:10.1182/blood.2019000095 (2019).

66 Schultz, K. R. et al. Improved early event-free survival with imatinib in Philadelphia chromosome-positive acute lymphoblastic leukemia: a children's oncology group study. Journal of clinical oncology : official journal of the American Society of Clinical Oncology 27, 5175-5181, doi:10.1200/JCO.2008.21.2514 (2009).

67 Gibbons, D. L. et al. Molecular dynamics reveal BCR-ABL1 polymutants as a unique mechanism of resistance to PAN-BCR-ABL1 kinase inhibitor therapy. Proceedings of the National Academy of Sciences of the United States of America 111, 3550-3555, doi:10.1073/pnas.1321173111 (2014). 
68 Ariad suspends ponatinib sales. Cancer discovery 4, 6-7, doi:10.1158/2159-8290.CDNB2013-163 (2014).

69 Blagosklonny, M. V., Toretsky, J. \& Neckers, L. Geldanamycin selectively destabilizes and conformationally alters mutated p53. Oncogene 11, 933-939 (1995).

70 Chavany, C. et al. p185erbB2 binds to GRP94 in vivo. Dissociation of the p185erbB2/GRP94 heterocomplex by benzoquinone ansamycins precedes depletion of p185erbB2. J Biol Chem 271, 4974-4977 (1996).

71 Stepanova, L., Leng, X., Parker, S. B. \& Harper, J. W. Mammalian p50Cdc37 is a protein kinase-targeting subunit of Hsp90 that binds and stabilizes Cdk4. Genes \& development 10, 1491-1502 (1996).

72 Soti, C., Racz, A. \& Csermely, P. A Nucleotide-dependent molecular switch controls ATP binding at the C-terminal domain of Hsp90. N-terminal nucleotide binding unmasks a C-terminal binding pocket. J Biol Chem 277, 7066-7075, doi:10.1074/jbc.M105568200 (2002).

73 Frieg, B., Gremer, L., Heise, H., Willbold, D. \& Gohlke, H. Binding modes of thioflavin $\mathrm{T}$ and Congo red to the fibril structure of amyloid-beta(1-42). Chemical communications 56, 7589-7592, doi:10.1039/d0cc01161d (2020).

74 Milic, D. et al. Recognition motif and mechanism of ripening inhibitory peptides in plant hormone receptor ETR1. Sci Rep 8, 3890, doi:10.1038/s41598-018-21952-3 (2018).

75 Gohlke, H. et al. Binding region of alanopine dehydrogenase predicted by unbiased molecular dynamics simulations of ligand diffusion. J Chem Inf Model 53, 2493-2498, doi:10.1021/ci400370y (2013).

76 Fontana, J. et al. Domain mapping studies reveal that the M domain of hsp90 serves as a molecular scaffold to regulate Akt-dependent phosphorylation of endothelial nitric oxide synthase and NO release. Circ Res 90, 866-873, doi:10.1161/01.res.0000016837.26733.be (2002).

77 Seidel, S. A. et al. Microscale thermophoresis quantifies biomolecular interactions under previously challenging conditions. Methods 59, 301-315, doi:10.1016/j.ymeth.2012.12.005 (2013).

78 Thulasiraman, V. \& Matts, R. L. Luciferase renaturation assays of chaperones and chaperone antagonists. Methods Mol Biol 102, 129-141, doi:10.1385/0-89603-5204:129 (1998).

79 Lo, M. C. et al. Evaluation of fluorescence-based thermal shift assays for hit identification in drug discovery. Anal Biochem 332, 153-159, doi:10.1016/j.ab.2004.04.031 (2004).

80 Schrödinger Release 2018-1: Maestro (Schrödinger, LLC, New York, NY, 2018).

81 Jorgensen, W. L., Chandrasekhar, J., Madura, J. D., Impey, R. W. \& Klein, M. L. Comparison of Simple Potential Functions for Simulating Liquid Water. J Chem Phys 79, 926-935, doi:Doi 10.1063/1.445869 (1983).

82 Martinez, L., Andrade, R., Birgin, E. G. \& Martinez, J. M. PACKMOL: A Package for Building Initial Configurations for Molecular Dynamics Simulations. Journal of Computational Chemistry 30, 2157-2164, doi:10.1002/jcc.21224 (2009).

83 Darden, T., York, D. \& Pedersen, L. Particle Mesh Ewald - an N.Log(N) Method for Ewald Sums in Large Systems. J Chem Phys 98, 10089-10092, doi:Doi 10.1063/1.464397 (1993).

84 Ryckaert, J. P., Ciccotti, G. \& Berendsen, H. J. C. Numerical-Integration of Cartesian Equations of Motion of a System with Constraints - Molecular-Dynamics of NAlkanes. J Comput Phys 23, 327-341, doi:Doi 10.1016/0021-9991(77)90098-5 (1977). 
85 Hopkins, C. W., Le Grand, S., Walker, R. C. \& Roitberg, A. E. Long-Time-Step Molecular Dynamics through Hydrogen Mass Repartitioning. J Chem Theory Comput 11, 1864-1874, doi:10.1021/ct5010406 (2015).

86 Roe, D. R. \& Cheatham, T. E. PTRAJ and CPPTRAJ: Software for processing and analysis of molecular. Dynamics Trajectory Data 9 (2013).

87 Homeyer, N. \& Gohlke, H. Free Energy Calculations by the Molecular Mechanics Poisson-Boltzmann Surface Area Method. Mol Inform 31, 114-122, doi:10.1002/minf.201100135 (2012).

88 Miller, B. R., 3rd et al. MMPBSA.py: An Efficient Program for End-State Free Energy Calculations. J Chem Theory Comput 8, 3314-3321, doi:10.1021/ct300418h (2012).

89 Onufriev, A., Bashford, D. \& Case, D. A. Exploring protein native states and largescale conformational changes with a modified generalized born model. Proteins $\mathbf{5 5}$, 383-394, doi:10.1002/prot.20033 (2004).

90 Manalastas-Cantos, K. et al. ATSAS 3.0: expanded functionality and new tools for small-angle scattering data analysis. J Appl Crystallogr 54, doi:doi:10.1107/S1600576720013412 (2021).

91 Svergun, D. I. Determination of the Regularization Parameter in Indirect-Transform Methods Using Perceptual Criteria. J Appl Crystallogr 25, 495-503, doi:Doi 10.1107/S0021889892001663 (1992).

92 Kozin, M. B. \& Svergun, D. I. Automated matching of high- and low-resolution structural models. J Appl Crystallogr 34, 33-41, doi:Doi $10.1107 / \mathrm{S} 0021889800014126$ (2001).

93 Mahon, F. X. et al. Selection and characterization of BCR-ABL positive cell lines with differential sensitivity to the tyrosine kinase inhibitor STI571: diverse mechanisms of resistance. Blood 96, 1070-1079 (2000). 


\section{Table of Content:}

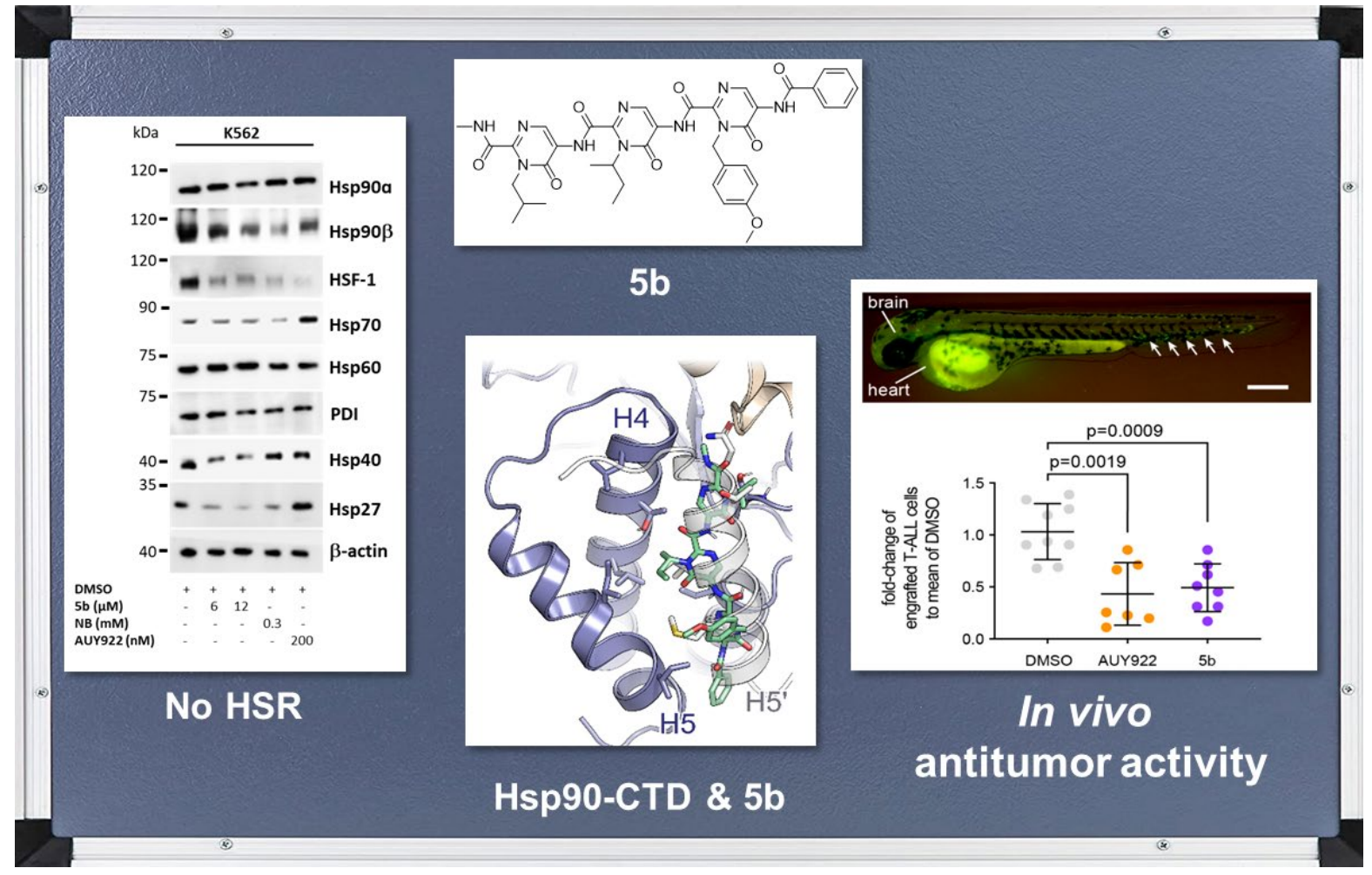

